\title{
Type I IFNs promote cancer cell stemness by triggering the epigenetic regulator KDM1B
}

Antonella Sistigu ( $\square$ antonella.sistigu@unicatt.it )

Università Cattolica del Sacro Cuore

Martina Musella

Università Cattolica del Sacro Cuore

Claudia Galassi

Università Cattolica del Sacro Cuore

Andrea Guarracino

University of Rome "Tor Vergata" https://orcid.org/0000-0001-9744-131X

Gwenola Manic

Italian Institute for Genomic Medicine, Candiolo Cancer Institute

Nicoletta Manduca

Università Cattolica del Sacro Cuore

Marco Pietrosanto

Università Tor Vergata

Manuela Helmer-Citterich

Università Tor Vergata

Matteo Pallocca

Regina Elena National Cancer Institute

Maurizio Fanciulli

Regina Elena National Cancer Institute

Anna Di Benedetto

Regina Elena National Cancer Institute

Cristiana Ercolani

Regina Elena National Cancer Institute

Edoardo Pescarmona

Regina Elena National Cancer Institute

\section{Laura Pizzuti}

Regina Elena National Cancer Institute

Francesca Sperati

Regina Elena National Cancer Institute

Michele Signore

Istituto Superiore di Sanità

Sara Vitale 
Università Cattolica del Sacro Cuore

\section{Giovanna Schiavoni}

Istituto Superiore di Sanità

\section{Fabrizio Mattei}

Istituto Superiore di Sanità

Adele De Ninno

Italian National Research Council

\section{Luca Businaro}

Italian National Research Council

Valeria Lucarini

Bambino Gesù Children's Hospital

\section{Laura Bracci}

Istituto Superiore di Sanità

\section{Eleonora Aricò}

Istituto Superiore di Sanità

\section{Giovanna Ziccheddu}

Regina Elena National Cancer Institute

\section{Francesco Facchiano}

Istituto Superiore di Sanità

\section{Stefania Rossi}

Istituto Superiore di Sanità

Massimo Sanchez

Istituto Superiore di Sanità

Alessandra Boe

Istituto Superiore di Sanità

\section{Mauro Biffoni}

Istituto Superiore di Sanità

\section{Ruggero De Maria ( $\sim$ ruggero.demaria@unicatt.it)}

Università Cattolica del Sacro Cuore

llio Vitale ( $\square$ ilio.vitale@iigm.it )

Italian Institute for Genomic Medicine, Candiolo Cancer Institute

\section{Article}

Keywords: viral mimicry, immunogenic cell death, epigenetic editing, demethylation, tumor-initiating cell death, immunotherapy

Posted Date: May 5th, 2021

DOl: https://doi.org/10.21203/rs.3.rs-436936/v1 
License: (c) (i) This work is licensed under a Creative Commons Attribution 4.0 International License. Read Full License

Version of Record: A version of this preprint was published at Nature Immunology on August 24th, 2022. See the published version at https://doi.org/10.1038/s41590-022-01290-3. 


\section{Abstract}

Cancer stem cells (CSCs) are immature, immortal cells within tumors, adept at resisting therapeutic pressure and responsible for local and distant disease recurrence. Non-genetic mechanisms of acquired resistance are increasingly being described, however molecular insights into this evolutionary process still lack. Here, we showed that Type I interferons (IFNs-I) act as molecular hubs of resistance during immunogenic chemotherapy, as they trigger the epigenetic regulator KDM1B, responsible for an adaptive, yet reversible, transcriptional rewiring of cancer cells towards stemness and immune escape. Accordingly, KDM1B pharmacological inhibition antagonizes the appearance of IFN-I-adapted CSCs, both in vitro and in vivo. Notably, IFN-I-adapted CSCs are heterogeneous in terms of multidrug resistance, plasticity, invasiveness and immunogenicity. Moreover, in breast cancer patients receiving anthracycline-based chemotherapy, IFN-I and KDM1B signatures positively correlate with CSC and immune evasion markers. Our study identifies IFN-I $\rightarrow$ KDM1B axis as a potent engine of cancer cell reprogramming and recommends KDM1B targeting an attractive adjunctive to immunogenic drugs to prevent CSC expansion and increase the long-term benefit of therapy.

\section{Introduction}

Despite the significant progress made over the last decades, the cure of cancer still remains one of the greatest medical challenges. Indeed, even neoplasms initially responding to conventional, targeted or immune-based therapies could acquire resistance and/or relapse into more aggressive and metastatic malignancies ${ }^{1,2}$. Therapy resistance occurs through a multifactorial, evolutionary process involving the acquisition of specific genetic and/or epigenetic alterations, which endow rare cancer cell variants with increased adaptiveness or fitness ${ }^{3}$. Like all evolutionary processes, cancer evolution is governed by the environment, and in particular by the changes inflicted by antitumoral treatments, which ultimately unleash tumor plasticity and generate evasive cell clones ${ }^{4,5,6,7}$.

Cancer stem cells (CSCs), also known as tumor-initiating or tumor-promoting cells, are the stem-like cell subpopulation within the tumor capable of self-renewal, multi-lineage differentiation, and responsible for tumor initiation, progression, metastatic spreading and therapy resistance $e^{8,9}$. Historically considered rare and quiescent, CSCs, in specific cancer types, have progressively been demonstrated to be relatively abundant, highly proliferating, and able to evolve over space and time leading to a high degree of genotypic, phenotypic and functional heterogeneity ${ }^{9,10}$. Moreover, non-CSC subsets can adapt to the changes of the tumor microenvironment (TME) by undergoing cell reprogramming and (re)generating CSCs $^{9}$.

Epigenetic dysregulations critically affect cancer-immune cell interactions and co-evolution during disease onset, progression and response to therapy by influencing cellular states and fates ${ }^{11}$. Not surprisingly given their role in normal stem cell maintenance, epigenetic mechanisms have also been involved in the preservation of $\mathrm{CSCs}^{12}$. This feature, together with the inherent reversibility of epigenetic 
modifications, makes the use of epigenome-targeting drugs (epidrugs) a unique opportunity to rationally target CSCs in combination with conventional therapies $11,13,14,15,16$.

One key concept in tumor immunology is that some chemotherapeutics, including (but not limited to) anthracyclines (e.g., doxorubicin, DOX), oxaliplatin (OXP) and cyclophosphamide ${ }^{17,18}$ induce cancer immunogenic cell death (ICD), a form of regulated cell death that initiates adaptive immune responses by the emission of damage-associated molecular patterns (DAMPs) ${ }^{19}, 20$. These include Type I interferons (IFNs-I), a family of cytokines that act as chemoattractants for T cells by triggering the production of the IFN-stimulated gene (ISG) C-X-C motif chemokine ligand 10 (CXCL10) upon binding to interferon alpha and beta receptor (IFNAR) ${ }^{18}$. Nonetheless, depending on the duration and intensity of the transduced signaling and/or the nature of the unleashed ISGs, IFNs-I can also display pro-tumorigenic effects ${ }^{21}$, promoting the expression of the immune checkpoint (IC) ligand CD274 (best known as PD-L1) ${ }^{18,22,23}$ and fostering cancer immunoediting ${ }^{24}$. Moreover, innate immune signaling upstream of IFNs-I has been associated with nuclear reprogramming and malignant transformation ${ }^{25}$.

In this work, we elucidated the downside of IFNs-I during ICD. Specifically, we showed that IFNs-I reprogram cancer cells toward a more aggressive, stem-like phenotype by recruiting the demethylase 1B (KDM1B), an epigenetic regulator also known as LSD2, which erases mono- and di-methyls on histone $\mathrm{H} 3$ at lysine 9 (H3K4me1 and H3K4me2) ${ }^{26}$. Such detrimental resetting represents a hitherto undescribed mechanism of tumor evolution, which drives acquired resistance and immune evasion. We term this process "regress to progress". Dissecting IFN-I dichotomy in cancer immune surveillance and escape offers key insights that could help develop rationale-based combinational strategies and increase the chances of success in cancer medicine.

\section{Results}

\section{Exogenous IFN-I administration promotes the enrichment and de novo induction of putative CSCs.}

To investigate the impact of the IFN-I $\rightarrow$ IFNAR axis on the appearance of cancer cells with a stem-like phenotype (hereafter referred to as CSCs), we selected a panel of cancer cell lines of distinct origin (epithelial or mesenchymal) and species (human or mouse) and treated them for $72 \mathrm{~h}$ with IFNs-I before assessing, by flow cytometry, the levels of prominin 1 (Prom1, best known as CD133), CD24 and CD44 surface markers, whose expression, alone and in combination, has been associated with putative CSCs. We observed that IFN-I exposure favors the enrichment of rare, CD $133^{+} \mathrm{CD} 24^{+} \mathrm{CD} 44^{+}$putative CSCs in all analyzed murine cancer cell lines. Specifically, we identified two main populations of IFN-I-enriched CSCs (IFN-CSCs) in MCA205 sarcoma cells: the CD133 ${ }^{+} \mathrm{CD} 24^{+} \mathrm{CD} 44^{+ \text {low }}$ (CD44L) and the $\mathrm{CD} 133^{+} \mathrm{CD} 24^{+} \mathrm{CD} 44^{\text {thigh }}(\mathrm{CD} 44 \mathrm{H}) \mathrm{CSC}$ subsets (Figure 1a). Putative IFN-CSC fractions were also detected in AT3 breast carcinoma (BC), namely the $\mathrm{CD} 133^{+} \mathrm{CD} 44^{+} \mathrm{CD} 24^{+ \text {low }}(\mathrm{CD} 24 \mathrm{~L})$ and $\mathrm{CD} 133^{+} \mathrm{CD} 44^{+} \mathrm{CD} 24^{\text {thigh }}$ (CD24H) CSC subsets, whereby we focused exclusively on the former, the widely recognized CSC subpopulation in $\mathrm{BC}^{27}$ (Figure 1a). Similarly, we found (I) $\mathrm{CD} 133^{+} \mathrm{CD} 44^{+} \mathrm{CD} 24^{+}$in CT26 colon carcinoma 
cell line and (ii) $\mathrm{CD} 133^{+} \mathrm{CD} 44^{+} \mathrm{CD} 24^{\text {low }}$ and $\mathrm{CD} 133^{+} \mathrm{CD} 44^{+} \mathrm{CD} 24^{\text {thigh }}$ in B16.F10 melanoma cell line (Supplementay Figure 1a). These results are in line with the intra- and inter-tumoral heterogeneity often ascribed to $\mathrm{CSCs}^{28}$. To assess whether this phenomenon was exclusive of the murine cancer model, we treated human U2OS osteosarcoma and MDA-MB-231 BC cell lines with recombinant human IFN-a2a and then analyzed the expression of standard human CSC markers. As expected, we detected IFN-CSC subpopulations in both U2OS (CD133 ${ }^{+} \mathrm{CD} 44^{+}$and $\mathrm{CD} 44 \mathrm{v} 6^{+} \mathrm{CD} 24^{+}$cell subsets) and MDA-MB-231 $\left(\mathrm{CD} 133^{+} \mathrm{CD} 44^{+}\right.$and $\mathrm{CD} 44 \mathrm{v} 6^{+} \mathrm{CD} 24^{+ \text {low }}$ cell subsets) cell lines (Supplementary Figure 1b).

We then investigated whether IFN-CSC enrichment relied on the positive selection of pre-existing CSCs or on an active de novo induction of CSCs, a strategy cancer cells might deploy to evolve resistance. To address this question, we isolated MCA205 $\mathrm{CD} 133^{+}$and CD133- (i.e., non-CSC) cell fractions by fluorescence-activated cell sorting (FACS) and treated them with IFNs-I. Intriguingly, by flow cytometry, we found that IFN-I treatment led to a significant increase in the fraction of CD44H cells and the levels of the pluripotency transcription factor (TF) SRY (sex determining region Y)-box 2 (SOX2) in both the CD133+ and CD133- subsets (Figure 1b). In parallel quantitative RT-PCR (qRT-PCR) analyses of common stemrelated TFs and CSC markers, we found that exogenous IFNs-I significantly upregulate Kruppel-like factor 4 (KIf4), POU domain, class 5, transcription factor 1 (Pou5f1, best known as Oct3/4), Sox2 and nestin (Nes) in FACS-isolated CD133" cells and Nanog homeobox (Nanog) in FACS-isolated CD133- and CD133 ${ }^{+}$ cells (Figure 1b). These results suggest the co-occurrence of a process of positive selection of rare, preexisting CSCs along with de novo CSC induction in response to exogenous IFNs-I.

We further analyzed the phenotypic and transcriptional profiles of IFN-CSCs, observing that IFN-I-treated epithelial cancer cells (AT3 and B16.F10) acquired a typical stem-like elongated morphology

(Supplementary Figure 1c). Moreover, in distinct murine cancer cells, IFNs-I promoted the emergence of the side population (SP, a bona fide CSC feature), which was significantly reduced by co-treatment with verapamil (VRP), a blocker of ATP-binding cassette (ABC) transporters (Figure 1c and Supplementary Figure 1d). Finally, IFN-I exposure induced significant upregulation of KIf4, Oct3/4, Sox2, Nanog, hes family bHLH transcription factor 1 (Hes 1) and Nes (Figure 1d and Supplementary Figure 1e), and endowed MCA205 and AT3 cancer cells with increased sphere forming ability (Figure 1e). Notably, only spheres pre-exposed to IFNs-I retained a CSC-related phenotypical and transcriptional profile when serially plated in standard CSC culture conditions (Figure 1f).

These data collectively demonstrate that exogenous IFNs-I favor the appearance of putative CSCs in multiple murine and human cancer cell lines.

\section{IFN-I signaling, during immunogenic chemotherapy, triggers cancer cell stemness.}

Since we have previously shown a key role for IFNs-I during bona fide ICD ${ }^{18}$, we asked whether CSC subpopulations could also be enriched during immunogenic chemotherapy. We took advantage of a library of pre-validated MCA-derived clones deficient for cardinal elements of the IFN-I pathway, including: (1) Ifnar1, (2) stimulator of interferon response cGAMP interactor 1 (Sting1, best known as Sting), (3) toll- 
like receptor 3 (T/r3), (4) toll-like receptor adaptor molecule 1 (Ticam1, best known as Trif), (5) interferon induced with helicase $\mathrm{C}$ domain 1 (Ifih 1, best known as Mda5), and (6) mitochondrial antiviral-signaling protein (Mavs, also known as Ips-1) (Figure 2a) ${ }^{18}$. We exposed these clones to the ICD inducer OXP ("donor" dying cells), then co-cultured "donor" dying cells with untreated clones of the same genotype ("receiving" viable cells) for $24 \mathrm{~h}$, and finally analyzed "receiving" cells at phenotypic and transcriptional levels (Supplementary Figure 2a). Similar to what observed upon IFN-I treatment, wild-type (Wt) clones responding to OXP displayed a significant increase of the two CD44H and CD44L CSC subpopulations (Figure 2b), which we will refer to as "ICD-CSCs". Notably, ICD-CSC enrichment was impaired only in Ifnar/- clones, indicating dependence on IFN-I signaling (Figure $2 \mathbf{b}$ ). Consistently, both IFN-I and OXP treatment induced the accumulation of CSC-related transcripts in Wt clones and (even if to a lesser and

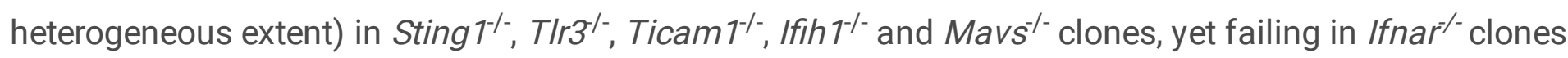
(Figure 2c). Similarly, the ICD inducer DOX favored a complete transcriptional rewiring toward pluripotency by enhancing the expression of the entire panel of reprogramming factors analyzed, while the non-ICD drug cisplatin (CDDP), which we previously shown to induce very low levels of IFNs ${ }^{18}$, affected the expression of only few transcriptional factors (Figure 2d). OXP also promoted the appearance of a SP in all murine cancer cell lines (Figure $\mathbf{2 e}$ and Supplementary Figure $\mathbf{2 b}$ ). Moreover, by exploiting DOX red fluorescence, we observed two distinct cell subsets ( $\mathrm{DOX}^{\text {+low }}$ and DOX ${ }^{\text {thigh }}$ ) in DOXtreated MCA205 cells, differing in their capability to extrude DOX as well as Hoechst 33342 (Figure $2 \mathbf{f}$ and Supplementary Figure 2c). Notably, following drug withdrawal, only DOX+low cells survived and resisted re-challenge with diverse ICD inducers (Supplementary Figure 2d), indicating their multidrug tolerance/resistance ${ }^{29}$. To explore the in vivo appearance of ICD-CSCs, we locally treated MCA205 tumors growing in syngeneic immunocompetent mice with DOX or CDDP and evaluated CSC enrichment in recollected xenografts 15 days post-treatment (i.e., when tumors start escaping growth control ${ }^{18}$ ). Strikingly, we found a twofold increase of $\mathrm{CD} 44 \mathrm{H}$ and $\mathrm{NANOG}^{+}$, upon DOX but not CDDP administration (Figure 2g).

Altogether, these results demonstrate that IFNs-I, during immunogenic chemotherapy, promotes CSC enrichment, both in vitro and in vivo, and point to this effect as an adaptive response cancer cells may deploy to escape therapy control.

\section{Horizontal transfer of nucleic acids from dying to viable cancer cells, upstream of IFN-I signaling, drives cancer stemness.}

To dissect the molecular mechanisms underlying ICD-CSC enrichment, we co-cultured OXP-treated "donor" MCA205 cells with untreated "receiving" MCA205 cells alone or in combination with benzonase (BNZase), which degrades all nucleic acids, or RNase A, RNase $\mathrm{H}$ or DNase, which selectively degrade ssRNAs, dsRNAs or DNA. We observed differential effects in the two CD44H and CD44L ICD-CSC subsets, with BNZase preventing the enrichment of both CSC populations, while RNase A, RNase $\mathrm{H}$ and DNase significantly affecting only CD44L cells (Figure 3a). Accordingly, BNZase halved the proportion of ICDCSCs in "receiving" AT3 and CT26 cells (Supplementary Figure 3a). The observation that only the 
depletion of all nucleic acids nullifies the enrichment of ICD-CSCs, again suggests that this phenomenon depends on an intact IFN-I signaling.

We next investigated the involvement of extracellular vesicles (EVs) in ICD-CSC enrichment. EVs isolated from "donor" MCA205 cells and stained with the non-toxic fluorescent membrane dye PKH26 were added to "receiving" MCA205 cells (Supplementary Figure 3b). EV uptake in "receiving" cells, confirmed by fluorescence microscopy and flow cytometry (Figure $3 \mathbf{b}$ ), induced a significant increase of $\mathrm{CD} 44 \mathrm{H}$ and CD44L CSC percentages and of the expression of most reprogramming factors, which were prevented by co-treatment with the actin inhibitor cytochalasin D (cyto D) (Figure 3c,d). Intriguingly, EVs from OXPtreated cancer cells carried mRNAs for reprogramming factors (KIf4, Myc, Oct3/4, Sox2, Nanog, Hes1, Nes), invasion molecules [snail family zinc finger 1 (Sna1, best known as Snail), Twist-related protein 1 (Twist1, also known as bHLHa38), Cadherin-2 (Cdh2, also known as $N$-cadherin/Ncad), Vimentin (Vim), and Fibronectin (Fn1)] and ICs, [Pdl1, programmed cell death 1 ligand 2 ( $P d c d 1 / g 2$, also known as PdI2), and lectin, galactose binding, soluble 9 ( $L$ gals 9 , best known as galectin-9) Figure 3e], suggesting their contribution to cancer cell de-differentiation and aggressiveness during immunogenic chemotherapy.

Altogether, these data indicate that ICD-CSC enrichment occurs through paracrine processes involving free and EV-mediated transfer of nucleic acids and stem-related mRNAs.

\section{IFN-CSCs and ICD-CSCs exhibit heterogeneity of drug-response, tumorigenic and invasive potential, and immunogenicity.}

We then analysed FACS-isolated CD44H and CD44L ICD-CSCs separately, and searched for hallmark CSC features like chemo-refractoriness, self-renewal ability, tumorigenic and metastatic potential, and the capability to escape immune control. We treated CD44H and CD44L cells with various ICD inducers, and found a diverse sensitivity to drugs, with only CD44H cells showing a higher therapeutic resistance than parental counterparts, both in vitro (Supplementary Figure 4a) and in vivo (Figure 4a), upon

transplantation in immunocompetent mice. On the one hand, by monitoring the in vitro evolution of FACSisolated MCA205 ICD-CSCs, we demonstrated as both subsets were able to rapidly regenerate the phenotypic complexity of parental cells (Figure 4b). On the other hand, in vivo studies revealed that CD44H ICD-CSCs are significantly more tumorigenic and less immunogenic than CD44L ICD-CSCs. Indeed, although both subpopulations were able to generate tumors in immunocompromised NOD SCID gamma (NSG) mice, only CD44H ICD-CSCs developed neoplasms at the lowest doses and overcame immunosurveillance thus growing in immunocompetent hosts at the highest number of injected cells (Figure 4c). Consistently, only half of the immunocompetent mice rejecting CD44H ICD-CSCs were vaccinated against viable parental cells (Figure 4d). Conversely, CD44L ICD-CSCs or parental MCA205 cells endowed animals with $100 \%$ long-term protection against tumor re-challenge. Moreover, when intravenously injected into immunocompetent mice, CD 44H (but not CD44L) ICD-CSCs developed lung metastases (Figure 4e).

Since we identified CD44H ICD-CSCs as the MCA205 subpopulation mainly driving in vivo tumor aggressiveness and therapeutic resistance, we focused on this subset. To gain insights into the 
immunogenicity of ICD-CSCs, we analyzed the proliferation rate of isolated $\mathrm{CD} 8^{+} \mathrm{H}-2 \mathrm{~Kb}$ /ovalbumin (OVA)-specific OT-1 T cells previously primed with dendritic cells (DCs) that had taken up apoptotic OVAexpressing CD44H (CD44H-OVA) ICD-CSCs or parental cells, and then boosted with viable cells of the same type. In line with the immune privileged nature observed in vivo (Figure 4c,d), CD44H-OVA ICD-CSCs induced a significantly lower expansion of OT-1 CD8 T cells than parental conterparts (Figure 5a) and resisted CD8-mediated killing (Figure 5b). These data prompted us to hypothesize that CD44H ICD-CSCs could escape immune control by inducing CD8 T cell exhaustion. To pursue this hypothesis, we analyzed the expression of common IC ligands, finding the upregulation of PDL1, PDCD1LG2, CEA1 and LGALS9 (Figure 5c). Consistently, CD8 ${ }^{+}$T tumor-infiltrating lymphocytes (TILs) isolated from MCA205-bearing mice 15 days after intratumoral injection of DOX (when CSC enrichment occurs), but not of CDDP (which does not enrich for CSCs), displayed a significant upregulation of the LGALS9 receptor IC Hepatitis A virus cellular receptor 2 (HAVCR2, best known as TIM-3) (Figure 5d). We extended the characterization of ICD-CSCs to AT3 cells (i.e., the CD24L cell subset), and confirmed their regenerative potential

(Supplementary Figure 4b) as well as the downregulation of the histocompatibility 2, K1 (H2-K1), and the upregulation of the IC ligands PDL1, PDCD1LG2 and LGALS9 (Figure 5c) and of the invasiveness markers Snail, Twist1, Ncad and Fn1 (Supplementary Figure 4c).

To further characterize ICD-CSC immunogenicity, we measured cytokine production through Luminex Multiplex Assay, observing a unique chemokine secretion pattern in CD44H MCA205 and CD24L AT3 ICDCSCs as compared to their respective parental cells. This encompasses reduced levels of proinflammatory chemokines CCL2 and CCL5, which mediate inflammatory monocyte trafficking and DCT cell interactions ${ }^{30}$, and enhanced capability to secrete CXCL1 and CXCL2 (the latter in CD24L AT3 cells), which promote chemoresistance and metastasis ${ }^{31}$ (Figure 5e). Notably, CD24L AT3 cells also showed higher levels of the regulatory T cell chemoattractant CCL $22^{32}$ than parental AT3 cells. Accordingly, when CD24L ICD-CSCs or parental AT3 cells were confronted with histocompatible splenocytes in ad hoc microfluidic devices ${ }^{33}$ and then analyzed by videomicroscopy for their in vitro capability to recruit immune cells, only parental cells were able to attract and stably interact with splenocytes at as early as $24 \mathrm{~h}$ (Figure 5f,g and Supplementary Movies 1-4). On the contrary, CD24L ICDCSCs failed to do so and, instead, migrated towards splenocytes starting a fleeting and unproductive interaction only upon 48h. Finally, when we confronted parental and CD24L AT3 cells in a microfluidic "competition" device (Supplementary Figure 4d), immune cells selectively migrated towards parental cells, moving away from CSCs (Figure $5 \mathrm{~h}, \mathrm{i})$.

Altogether, these results allowed us to make several key observations: adaptation of cancer cells to immunogenic chemotherapy enables cell selection and drives phenotype switching. Both phenomena actively contribute to intratumor heterogeneity as the collection of CSC subpopulations have differential therapeutic response, aggressiveness and immunogenicity.

\section{Global chromatin remodeling downstream of IFNs-I.}


To dissect the mechanisms underlying cancer cell reprogramming downstream of IFNs-l, we mapped the chromatin landscape of parental $(\mathrm{P})$ and $\mathrm{CD} 44 \mathrm{H}(\mathrm{H})$ MCA205 cells by the assay for transposaseaccessible chromatin using sequencing (ATAC-seq) (Figure 6a-c). By analyzing ATAC-seq peaks, we conceived a closed-to-open $(C \rightarrow O)$ and an open-to-closed $(O \rightarrow C)$ logic, and stratified genes in 4 groups.

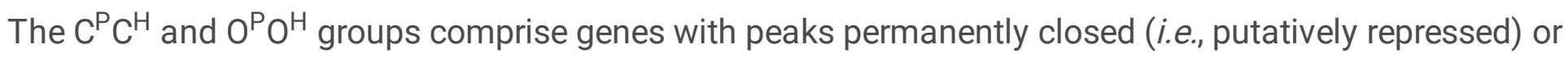
open (i.e., putatively expressed) in both samples, while the $\mathrm{C}^{\mathrm{P}} \mathrm{O}^{\mathrm{H}}$ and $\mathrm{O}^{\mathrm{P}} \mathrm{C}^{\mathrm{H}}$ groups comprise genes whose peaks are closed in parental cells and open in CD44H IFN-CSCs and viceversa. In particular, we focused on the $\mathrm{C}^{\mathrm{P}} \mathrm{O}^{\mathrm{H}}$ group containing genes putatively more expressed in CSCs. Intriguingly, this group encompasses genes involved in cancer stemness (Myc and Sox), embryonic development (Tbx4), epithelial-to-mesenchymal transition (EMT) (Gata6 and Tfcp2), cancer cell invasiveness and metastatization (Myct1, Spire1 and Trpm4), tumorigenesis, tumor progression and therapy resistance (Slc6a6, Baiap2, TtII7 and Wee1), the negative regulator of the antigen presentation machinery (APM) Gpr17, and the inhibitor of granzyme activity Serpin (Figure 6a). Consistently, in the $\mathrm{O}^{\mathrm{P}} \mathrm{C}^{\mathrm{H}}$ group we found tumor suppressor genes (Cdh, Cdk2ap1, Dlg2, Ripk3 and Fbxw2) and genes controlling tumor growth (Mtor and Ncam1), APM functionality (Tap1, Tap2 and CtsI) and inflammation (II24, II27, Gsdmd and Uba7) (Figure 6a). Subsequent integration with RNA-seq analyses confirmed an increased expression of genes involved in tumorigenesis, tumor progression, invasiveness (e.g., Csf1r, Trpm4, Itga5, Wee1, Baiap2, TtII7 and Spire1) and immune escape (e.g., Gpr17), coupled with repression of genes involved in tumor suppression and immune recognition (like Cdh1, I/12b, T/r5, Cdk2ap1, I/34, //16 and Cts/) in CD44H IFNCSCs, as compared to parental cells (Figure 6d).

We next inferred and reconstructed protein-protein interaction subnetworks and biological processes specifically modulated in CD44H IFN-CSCs by using the clusterProfiler and enrichPlot R packages (Figure 6e and Supplementary Figure 5a). Gene ontology (GO) analysis showed that most of the upregulated genes in $\mathrm{CD} 44 \mathrm{H}$ cells (red module) have significant functional connections with cell growth promotion, stemness maintenance and tissue remodeling, with immune suppression (e.g., negative regulation of leukocyte activation), despite an intact response to IFN-I and IFN-II, with response to stress (e.g., positive regulation of response to DNA damage stimulus, regulation of autophagy and apoptosis), lipid metabolism, and, of note, with enhanced chromatin accessibility. Accordingly, we organized the downregulated genes (blue module) into 4 biological processes: cell growth arrest, cell differentiation, oxidative phosphorylation and protein dephosphorylation. These results provide clues about the modular re-organization of specific pathways downstream of IFNs-I.

Of note, among the genes specific of the CSC fraction (CD44H cells), we also identified multiple ISGs, including (but not limited to) Ifi27/2a, Ifi27/2b and the epigenetic regulator $K d m 1 b$ (Figure 6a,b). We were particularly intrigued by $K d m 1 b$ since chromatin remodeling plays a critical role in cancer evolution, cellular plasticity and immune escape ${ }^{12,34,35,36}$. At first, we measured the enrichment of TF-binding motifs in the ATAC-seq study by using the HOMER motif software (Figure $6 \mathrm{c}$ and Supplementary Figure 5b). We observed significant differences between CSCs and parental cells, in particular we found enrichment of motifs for various TFs of the helix-turn-helix (HTH) superfamily (i.e., RFX, Rfx1, Rfx2, Rfx5 
and X-box), the Homeobox basic helix-loop-helix (bHLH) member Pitx1:Ebox, the Rel homology domain (RHD) family member NFkB-p65, and the zinc finger (Zf) family member ZBTB in CD44H cells, suggesting their major role in the global chromatin remodeling in CSCs. Conversely, the $\mathrm{Zf}$ motifs CTCF, BORIS and NRSF, the transcriptional enhanced associate domain (TEA, TEAD) motifs (i.e., TEAD and TEAD1-4), the Rel homology domain (RHD)-basic leucine-zipper (bZIP) superfamily member NFAT-AP1, the ETS, RUNT, the interferon-sensitive response element (ISRE) and the CCAAT box-binding transcription factor (CTF) motifs were more accessible in parental cells. Thereafter, we explored the role of the ISG KDM1B in the induction of ICD-CSCs. To this aim, we added the KDM1B inhibitor tranylcypromine (TCP) to the "donor"-"receiving" in vitro co-culture and found a significant reduction of $\mathrm{CD} 44 \mathrm{H}$ percentages in "receiving" cells (Figure 6f). Of note, in vivo administration of TCP in MCA205 tumor bearing mice (Figure $6 \mathrm{~g}$, left panel), prevented the enrichment of ICD-CSCs as well as the induced expression of TIM-3 on CD8 ${ }^{+}$ TILs (Figure 6g, central and right panels).

Overall, these data demonstrate that KDM1B, downstream of IFNs-I, edits the epigenome of cancer cells toward stemness, immune escape and therapy resistance (Figure $6 \mathrm{~h}$ ).

\section{IFN-I metagenes correlate with stemness in BC patients.}

To investigate the clinical relevance of the IFN-I $\rightarrow$ KDM1B axis, we first calculated the Spearman correlation between $K D M 1 B$, IFN-I-related metagenes, stem-related reprogramming factors and IC ligands in multiple publicly available transcriptomic data of $B C$ patients responsive to anthracyclines ${ }^{18,37}$. We observed that the expression levels of KDM1B significantly correlated with those of MYC and POU5F1 in two out of three available datasets and of IFNB1, CXCL10, and CD274/PD-L1 in at least one dataset (Figure 7a). Along similar lines, IFNB1 expression positively correlated with the expression of SOX2 in half of the analyzed datasets, of KLF4, NES and PDCD1LG2/PD-L2 in one dataset, and of POU5F1 and NANOG in two datasets (Figure 7a and Supplementary Figure 6a). Similarly, the ISGs MX1, CXCL 10, DHX58, OASL and STAT1 correlated with the stemness factors MYC, POU5F1, SOX2 and NANOG, and with the IC ligands $P D C D 1 \angle G 2 / P D-L 2$ and $C D 274 / P D-L 1$, these last in turn correlating with KLF4, MYC, POU5F1, SOX2 and NANOG, in at least one dataset (Figure 7a and Supplementary Figure 6a). Next, we selected a BC cohort including 1902 patients on the online biomarker validation tool Metabric (Figure $7 \mathbf{b}, \mathbf{c}$ and Supplementary Figure $6 \mathrm{~b}-\mathrm{e}$ ) and performed multivariate survival analysis by stratifying patients into two groups, according to risk behavior. In details, patients were assigned to either the high-risk or the low-risk group based on the high and low expression, respectively, of gene pairs selected among KDM1B-related, IFN-I-related and stem-related factors (KDM1B-SOX2, MX1-SOX2, CXCL10-SOX2, and OASL-SOX2). Of note, high-risk group patients exhibited a significantly reduced distant and local recurrence-free incidences (Figure $7 \mathbf{b}$ and Supplementary Figure $\mathbf{6 b - d}$ ) and, accordingly, disease-specific survival was decreased if compared to low-risk group patients (Figure 7c and Supplementary Figure 6e).

To further correlate IFN-I and CSC signatures, we performed longitudinal immunohistochemistry (IHC) analyses on consecutive formalin fixed paraffin-embedded $B C$ biopsies, assessing the expression of MX1 and CD44-CD24 on CD45 cancer cells at pre- (T0; at diagnosis) and post- (T1, at surgery) neo-adjuvant 
anthracycline-based chemotherapy (Figure 7d-f and Supplementary Table 1). We found a significant increase of $\mathrm{CD} 44^{+} \mathrm{CD} 24^{-/ \text {low }}$ Allred scores in $15 \%$ of cases (Figure 7f). Altogether, these results suggest the co-occurrence of IFN-I signature and CSC markers during anthracycline-based immunogenic chemotherapy.

\section{Discussion}

IFNs-I may either restrain or promote tumor growth depending on the duration and intensity of the transduced signaling, two features that jointly delineate the patterns of ISG expression, so-called "IFN signature" 21 , and shape the accessibility to chromatin, so-called "IFN-mediated epigenomic signature" 38 , 39 . The leverage of transcriptional and epigenetic changes defines cell responses to environmental hints and dictates the efficacy of natural and therapy-induced immunosurveillance ${ }^{11,17,40,41}$. Here, we provide preclinical and clinical evidence that the acute induction of IFNs-I, as during chemotherapy-induced ICD, favors the appearance of subpopulations of CSCs. This occurs via positive selection of CSCs coupled to a KDM1B-dependent de novo reprogramming of cancer cells toward a stem-like phenotype. Therefore, beyond stimulating antitumor immune response, IFNs-I can foster malignant progression leaving a detrimental "imprint" on cancer cells.

Our study sheds light on the debated and poorly investigated contribution of IFN-I signaling on tumor heterogeneity and CSC induction. On the one hand, we and others previously reported that IFNs-I hinder CSC generation/survival, showing that the abrogation of steady-state endogenous IFN-I signaling leads to the emergence of breast CSCs in HER2/neu transgenic mice and triple-negative $\mathrm{BC}^{42,43}$. On the other hand, acute production or exogenous administration of IFNs-I favored cancer stemness in mouse models of pancreatic cancer ${ }^{44}$ and human BC and squamous carcinoma cell lines ${ }^{45}$. Nonetheless, in these studies the molecular mechanisms underlying IFN-I-CSC expansion have not been analyzed, and this phenomenon has been neither investigated in the context of ICD, nor associated with potential cancer cell reprogramming. In this respect, it appears of interest the evidence that the induction of the ISG IFI27 in ovarian carcinoma biopsies and cell lines drives EMT, cancer stemness, invasiveness and therapeutic resistance ${ }^{46}$. Whether IFI27 is involved in ICD-CSC expansion requires further investigations. Based on our results, we surmise that, when produced at chronic low levels, IFNs-I limit CSC proliferation and survival, restraining tumor growth. At odds, acute IFN-I production, as during immunogenic chemotherapy, favors the survival of pre-existing CSCs and cancer cell de-differentiation, potentially leading to therapy resistance/failure.

Here, we also found a certain degree of phenotypic and functional heterogeneity within IFN-CSCs, consistently with the current view of an adaptable, evolutive and dynamic nature of $\operatorname{CSCs}^{47,48}$. Moreover, we showed that specific IFN-I-CSC subsets are characterized by high resistance to (immuno)chemotherapy and in vivo tumorigenicity, metastatic potential and low immunogenicity, in line with previous observations ${ }^{49,50}$. In our setting, CSC immunoprivilege encompasses a reduced capability of these cells to attract and stably interact with effector immune cells, in part due to decreased secretion 
of proinflammatory chemokines and enhanced capability to suppress $\mathrm{T}$ cell activation, and in part due to upregulated expression of IC ligands and cognate receptors. At the mechanistic level, IFN-I-related immune escape has been previously associated with the upregulation in cancer (stem) cell of (i) PD-L1 and LGALS9 ${ }^{23}$, (ii) nitric oxide synthase 2 (NOS2), which favors the recruitment of regulatory cells ${ }^{51}$, and (iii) Serpinb9, which inhibits granzyme B activity and thus $C D 8^{+} \mathrm{T}$ cell cytotoxicity ${ }^{52}$. Intriguingly, by integrating ATAC-seq and RNA-seq data, we found upregulation, in CD44H IFN-CSCs, of serpins and downregulation of Uba7, a tumor suppressor ISG which codes for a protein able to attract effector $T$ cells ${ }^{53}$. Whether these factors play a major role in protecting CSC from immune attack remains to be established.

By co-culturing experiments, we demonstrated that ICD-CSC enrichment involves an autocrine/paracrine cancer cell-to-cancer cell circuitry centered on the IFN-I $\rightarrow$ IFNAR $\rightarrow$ KDM1B signaling pathway. We propose a model whereby CSC induction lies on the horizontal transfer of nucleic acids and possibly stem-related encoding mRNAs from cancer cells undergoing ICD to viable cancer cells. Notably, such intercellular communication can also occur via EVs, according to the role recently ascribed to EVs in conferring resistance and metastatic recurrence to anthracyclines ${ }^{54}$. In our model, once transferred from dying to viable cells, nucleic acids act as DAMPs leading to acute IFN-I production, which ultimaltely drives KDM1B-mediated cancer cell reprogramming, and thus therapy failure and tumor re-growth (Figure $6 \mathbf{h}$ ). Two results support this IFN-I-KDM1B centered model. First, ICD-CSC enrichment was abrogated by inhibiting the ISG KDM1B, by ablating IFNAR signaling, or by depleting the entire (but not single) spectrum of self-nucleic acids. This latter result also suggests the existence of a certain degree of redundancy in, and compensation between, nucleic acid-sensing pathways, ensuring IFN-I production also when specific signals or sensors are depleted/missing. Second, by integrating ATAC-seq and RNAseq analyses, we revealed extensive transcriptional reprogramming in ICD-CSCs, manifested with increased expression of genes related to stemness, invasiveness and metastatization coupled to silencing of tumor suppressor and immune stimulating genes.

Although further confirmation in human models is required, we hypothesize that activation of the IFN-I signaling directly stimulates CSCs in tumors undergoing ICD. We thus surmise the existence of a mechanism similar to that underlying virus-induced cell transdifferentiation which leads to the upregulation of core pluripotency genes ${ }^{25}$. Supporting our hypothesis, IFNs-I were recently ascribed to have a role in chromatin remodeling and gene expression reprogramming ${ }^{39}$, 55 . Moreover, the expression of diverse KDMs has been correlated with "cold" TMEs in different tumor models, as also the use of epidrugs with the reinstatement of inflammation ${ }^{56,57,58}$. Recently, epidrug-related immune modulation was shown to co-occur with MYC suppression ${ }^{59}$. Moreover, our retrospective studies on BC patients that had received anthracycline based therapy showed signs of mutual correlation between KDM1B, IFN-Irelated, stem-associated and IC metagenes, as well as the enrichment of CSCs along with immunogenic treatments. Further validation on a larger cohort of patients with patient follow-up will be launched. 
In conclusion, we demonstrated that acute IFNs-I elicit a protective but ephemeral anticancer response. By triggering the ISG KDM1B, IFNs-I promote the appearance of heterogeneous CSCs, including CSCs with traits of immune privilege and therapy resistance. This evidence provides the basis for the use of epidrugs as adjunctives to anticancer immunogenic therapies, including conventional chemotherapies and current and upcoming immunotherapies, as a therapeutical means to prevent CSC expansion and patrol tumor recurrence.

\section{Abbreviations}

ABC, ATP-binding cassette; APM, antigen presentation machinery; ATAC-seq, assay for transposaseaccessible chromatin using sequencing; ATCC, American Type Culture Collection; BC, breast cancer; BNZase, benzonase nuclease; $\mathrm{CD} 44 \mathrm{H}, \mathrm{CD} 133^{+} \mathrm{CD} 24^{+} \mathrm{CD} 44^{\text {+high }} ; \mathrm{CD} 44 \mathrm{~L}, \mathrm{CD} 133^{+} \mathrm{CD} 24^{+} \mathrm{CD} 44^{\text {+low }}$; CDDP, cisplatin; CSCs, cancer stem cells; cyto D, cytochalasin D; DAMPs, damage-associated molecular patterns; DOX, doxorubicin; DRFI, distant relapse-free incidence; DSS, disease-specific survival; EMT, epithelial-tomesenchymal transition; EVs, extracellular vesicles; FACS, fluorescence activated cell sorting; IC, immune checkpoint; ICD, immunogenic cell death; ICD-CSCs, immunogenic cell death-induced CSCs; IFNs-I, Type I interferons; IFN-CSCs, Type I interferon-induced CSCs; IHC, immunohistochemistry; ISGs, interferonstimulated genes; LRFI, local relapse-free incidence; MDR, multidrug resistance; MTX, mitoxantrone dihydrochloride; NSG, NOD SCID gamma; OVA, ovalbumin; OXP, oxaliplatin; PI, propidium iodide; qRT-PCR, quantitative RT-PCR; SP, side population; TCP, tranylcypromine; TF, transcription factor; TILs, tumor infiltrating lymphocytes; TME, tumor microenvironment; VRP, verapamil.

\section{Declarations}

Acknowledgments: We thank Paola Di Matteo, Roberto Ricci, Massimo Spada and Daniele Macchia for technical assistance, Dr. Enrico Proietti and Dr. Paola Sestili for providing IFNs-I, Pr. Laurence Zitvogel for providing MCA-derived clones, Dr. Oliver Kepp and Dr. Shuai Zhang for providing MCA-OVA, Dr. Ivan Tattoli for language and grammar editing. In vivo experiments were performed at Plaisant Castel Romano and Istituto Superiore di Sanità (Rome, Italy). This work was supported by the Associazione Italiana per la Ricerca sul Cancro (AIRC, Start-Up 2016 \#18418) and Ministero Italiano della Salute (RF_GR-201302357273). IV is supported by the AIRC (IG 2017 grant number 20417) and a startup grant from the Italian Institute for Genomic Medicine (Candiolo, Turin, Italy) and Compagnia di San Paolo (Turin, Italy). GS is supported by the AIRC (IG 2018 grant number 21366). MHC is supported by the AIRC (IG 2019 grant number 16895). RDM is supported by the AIRC (5 × 1000 grant number 9979) and Ministero Italiano della Salute (RF_RF-2018-12367044)

Author Contributions: M.M. designed and performed experiments, analyzed and interpreted data and wrote the manuscript with the help of C.G., G.M., N.M.; A.G., M.S., M.P., M.P., M.F., M.H.C. analyzed data and performed bioinformatic studies; F.S. performed statistical analysis; A.D.B., C.E., E.P. performed IHC experiments and analysis; L.P. provided clinical data; A.D.N., L.B. designed and realized microfluidic systems; G.S., F.M., V.L. performed and analyzed experiments on microfluidic devices, F.F., S.R., G.Z. 
performed and analyzed Luminex assay, M.S., A.B. analyzed flow cytometry data; L.B., E.A., S.V., M.B. designed experiments, I.V., A.S. obtained funding, supervised the project, designed experiments, analyzed data and wrote the manuscript; R.D.M. obtained funding, supervised the project, designed experiments and wrote the manuscript.

Author Disclosure: The authors have no conflicts of interest to disclose.

\section{Methods}

\section{KEY RESOURCES TABLE}




\begin{tabular}{|c|c|c|}
\hline REAGENT or RESOURCE & SOURCE & IDENTIFIER \\
\hline \multicolumn{3}{|l|}{ Antibodies } \\
\hline Rat monoclonal anti-CD133 (13A4) & eBioscience $^{\mathrm{TM}}$ & $\begin{array}{l}\text { Cat\# 17-1331-81, } \\
\text { RRID:AB_823120 }\end{array}$ \\
\hline Rat monoclonal anti-CD24 (M1/69) & eBioscience $^{\mathrm{TM}}$ & $\begin{array}{l}\text { Cat\# 12-0242-82, } \\
\text { RRID:AB_465602 }\end{array}$ \\
\hline Rat monoclonal anti-CD44 (IM7) & eBioscience $^{\mathrm{TM}}$ & $\begin{array}{l}\text { Cat\# 11-0441-82, } \\
\text { RRID:AB_465045 }\end{array}$ \\
\hline Rat monoclonal anti-CD8a (53-6.7) & eBioscience $^{\mathrm{TM}}$ & $\begin{array}{l}\text { Cat\# 17-0081-82, } \\
\text { RRID:AB_469335 }\end{array}$ \\
\hline Rat monoclonal anti-CD273 (122) & eBioscience $^{\mathrm{TM}}$ & $\begin{array}{l}\text { Cat\# 11-9972-81, } \\
\text { RRID:AB_465461 }\end{array}$ \\
\hline Mouse monoclonal anti-CD66a (CC1) & eBioscience $^{\mathrm{TM}}$ & $\begin{array}{l}\text { Cat\# 12-0661-80, } \\
\text { RRID:AB_1311201 }\end{array}$ \\
\hline Mouse monoclonal anti-H2-K1 (AF6-88.5.5.3) & eBioscience $^{\mathrm{TM}}$ & $\begin{array}{l}\text { Cat\# 11-5958-80, } \\
\text { RRID:AB_11151335 }\end{array}$ \\
\hline Rat monoclonal anti-CD274 (10F.9G2) & BioLegend $^{\circledR}$ & $\begin{array}{l}\text { Cat\# 124312, } \\
\text { RRID:AB_10612741 }\end{array}$ \\
\hline Rat monoclonal anti-Galectin-9 (108A2) & BioLegend ${ }^{\circledR}$ & $\begin{array}{l}\text { Cat\# 137903, } \\
\text { RRID:AB_10568785 }\end{array}$ \\
\hline Rat monoclonal anti-CD366 (RMT3-23) & eBioscience $^{\mathrm{TM}}$ & $\begin{array}{l}\text { Cat\# 11-5870-82, } \\
\text { RRID:AB_2688129 }\end{array}$ \\
\hline Rat monoclonal anti-CD45 (30-F11) & eBioscience $^{\mathrm{TM}}$ & $\begin{array}{l}\text { Cat\# MCD4528, } \\
\text { RRID:AB_10373710 }\end{array}$ \\
\hline Mouse monoclonal anti-CD133/1 (AC133) & Miltenyi Biotec & Cat\# 130-113-106 \\
\hline Recombinant monoclonal anti-CD44 (REA690) & Miltenyi Biotec & Cat\# 130-113-342 \\
\hline Mouse monoclonal anti-CD24 (ML5) & BD Biosciences & $\begin{array}{l}\text { Cat\# BBA13, } \\
\text { RRID:AB_356935 }\end{array}$ \\
\hline Mouse monoclonal anti-CD44v6 (2F10) & R\&D Systems & $\begin{array}{l}\text { Cat\# BBA13, } \\
\text { RRID:AB_356935 }\end{array}$ \\
\hline Rabbit polyclonal anti-MX1 & Sigma-Aldrich & $\begin{array}{l}\text { Cat\# HPA049724, } \\
\text { RRID:AB_2680862 }\end{array}$ \\
\hline Rabbit monoclonal anti-CD44 (SP37) & Sigma-Aldrich & Cat\# SAB5500068 \\
\hline Mouse monoclonal anti-CD24 (SN3b) & Agilent Technologies & $\begin{array}{l}\text { Cat\# CBL561, } \\
\text { RRID:AB_11212454 }\end{array}$ \\
\hline Mouse monoclonal anti-CD45 (2B11+PD7/26) & Agilent Technologies & $\begin{array}{l}\text { Cat\# GA75161-2, } \\
\text { RRID:AB_2661839 }\end{array}$ \\
\hline Goat aniti-mouse Alexa Fluor ${ }^{\circledR}$ Plus 488 & Thermo Scientific & Cat\# A32723 \\
\hline \multicolumn{3}{|l|}{ Chemicals, Peptides, and Recombinant Proteins } \\
\hline Purified mouse IFN- $\alpha / \beta$ and mock & $\begin{array}{l}\text { Dr. E. Proietti, Istituto } \\
\text { Superiore di Sanità, Rome }\end{array}$ & \\
\hline Recombinant human Roferon-A ${ }^{\circledR}$ & Hoffmann-La Roche Ltd & \\
\hline Cisplatin & Sigma-Aldrich & Cat\# P4394 \\
\hline Doxorubicin hydrochloride & Sigma-Aldrich & Cat\# D1515 \\
\hline Mitoxantrone dihydrochloride & Sigma-Aldrich & Cat\# M6545 \\
\hline Oxaliplatin & Selleck Chemicals & Cat\# S1224 \\
\hline Cytochalasin D & Sigma-Aldrich & Cat\# C8273 \\
\hline Tranylcypromine & Calbiochem & Cat\# 616431 \\
\hline Verapamil & Sigma-Aldrich & Cat\# V4629 \\
\hline Benzonase nuclease & Sigma-Aldrich & Cat\# E8263 \\
\hline DNase I & Qiagen & Cat\# 79254 \\
\hline
\end{tabular}




\begin{tabular}{|c|c|c|}
\hline Ribonuclease A & Sigma-Aldrich & Cat\# R6513 \\
\hline Ribonuclease $\mathrm{H}$ & Thermo Scientific & Cat\# EN0201 \\
\hline Collagenase A & Sigma-Aldrich & Cat\# COLLA-RO \\
\hline Trypan Blue Stain (0.4\%) & Thermo Scientific & Cat\# 15250061 \\
\hline DMSO & Sigma-Aldrich & Cat\# 5879 \\
\hline Hoechst 33342 & Thermo Scientific & Cat\# H1399 \\
\hline DAPI & Thermo Scientific & Cat\# D1306 \\
\hline PI & Thermo Scientific & Cat\# P1304MP \\
\hline CFSE & Sigma-Aldrich & Cat\# 21888 \\
\hline SeaPlaque GTG Agarose & Lonza & Cat\# 50111 \\
\hline Ppia (Mm00620857_s1) primers & Applied Biosystems $^{\text {TM }}$ & Cat\# 4331182 \\
\hline Klf4 (Mm00516104_m1) primers & Applied Biosystems $^{\text {TM }}$ & Cat\# 4331182 \\
\hline Myc (Mm00487804_m1) primers & Applied Biosystems $^{\text {TM }}$ & Cat\# 4331182 \\
\hline Pou5f1 (Mm03053917_g1) primers & Applied Biosystems $^{\text {TM }}$ & Cat\# 4331182 \\
\hline Sox2 (Mm03053810_s1) primers & Applied Biosystems $^{\text {TM }}$ & Cat\# 4331182 \\
\hline Nanog (Mm02019550_s1) primers & Applied Biosystems ${ }^{\mathrm{TM}}$ & Cat\# 4331182 \\
\hline Hes1 (Mm01342805_m1) primers & Applied Biosystems $^{\text {TM }}$ & Cat\# 4331182 \\
\hline Nes (Mm00450205_m1) primers & Applied Biosystems $^{\text {TM }}$ & Cat\# 4331182 \\
\hline Twist1 (Mm00442036_m1) primers & Applied Biosystems $^{\text {TM }}$ & Cat\# 4331182 \\
\hline Snai1 (Mm00441533_g1) primers & Applied Biosystems ${ }^{\mathrm{TM}}$ & Cat\# 4331182 \\
\hline Cdh2 (Mm01162497_m1) primers & Applied Biosystems $^{\text {TM }}$ & Cat\# 4331182 \\
\hline Vim (Mm00619195_g1) primers & Applied Biosystems $^{\text {TM }}$ & Cat\# 4331182 \\
\hline Fn1 (Mm01256744_m1) primers & Applied Biosystems $^{\text {TM }}$ & Cat\# 4331182 \\
\hline Lgals9 (Mm00495295_m1) primers & Applied Biosystems ${ }^{\mathrm{TM}}$ & Cat\# 4331182 \\
\hline Pdcd1lg2 (Mm00451734_m1) primers & Applied Biosystems ${ }^{\mathrm{TM}}$ & Cat\# 4331182 \\
\hline $\begin{array}{l}P d l 1 \text { (fw: GCATTATATTCACAGCCTGC, rw: } \\
\text { CCCTTCAAAAGCTGGTCCTT) primers }\end{array}$ & Sigma-Aldrich & Custom \\
\hline Agarose gel & Sigma-Aldrich & Cat\# A9539 \\
\hline SYBR Safe DNA gel stain & Thermo Scientific & Cat\# S33102 \\
\hline
\end{tabular}

\begin{tabular}{|l|l|l|}
\hline Crystal Violet & Sigma-Aldrich & Cat\# C0775 \\
\hline Matrigel & BD Biosciences & Cat\# 354234 \\
\hline PKH26 Red Fluorescent Cell Linker & Sigma-Aldrich & Cat\# PKH26GL \\
\hline CD45 MicroBeads & Miltenyi & Cat\# 130-052-301 \\
\hline Agencourt AMPure SPRI beads & Beckman Coulter & Cat\# A63880 \\
\hline
\end{tabular}




\section{Critical Commercial Assays}

\begin{tabular}{|c|c|c|}
\hline RNeasy Plus Mini Kit & Qiagen & Cat\# 74134 \\
\hline RNeasy Mini Kit & Qiagen & Cat\# 74104 \\
\hline GoTaq ${ }^{\circledR}$ Probe 1-Step RT-qPCR System & Promega & Cat\# A6120 \\
\hline $\begin{array}{l}\text { Mouse magnetic Luminex screening assay } \\
\text { CCL11/Eotaxin (BR74), CCL12/MCP-5 (BR42), } \\
\text { CCL2/MCP-1/JE (BR18), CCL3/MIP-1 } \alpha \text { (BR46), } \\
\text { CCL4/MIP-1ß (BR51), CCL22/MDC (BR75), CCL8/MCP- } \\
2 \text { (BR38), CXCL1/KC (BR13), CXCL10/IP-10 (BR37), } \\
\text { CXCL12/SDF-1 } \alpha \text { (BR54), CXCL2/MIP-2 (BR20) }\end{array}$ & R\&D Systems & Cat\# LXSAMSM-12 \\
\hline $\begin{array}{l}\text { Mouse pluripotent stem cell transcription factor analysis } \\
\text { kit assayssing Nanog (M55-312) }\end{array}$ & BD Biosciences & Cat\# 560585 \\
\hline CellTiter-Glo ${ }^{\circledR}$ Luminescent Cell Viability Assay & Promega & Cat\# G7572 \\
\hline ExoEasy Maxi Kit & Qiagen & Cat\# 76064 \\
\hline Nextera Library Prep Kit & Illumina & Cat\# 20018704 \\
\hline MinElute PCR purification kit & Qiagen & Cat\# 28004 \\
\hline KAPA Library Quantification Kit for Illumina platforms & $\begin{array}{l}\text { KAPA } \\
\text { Biosystems - } \\
\text { Hoffmann - La } \\
\text { Roche } \\
\end{array}$ & Cat\# KR0405 \\
\hline TruSeq Stranded mRNA Library kit & Illumina & Cat\# 20020594 \\
\hline ChromoPlex TM1 Dual Detection & $\begin{array}{l}\text { Bond III, Leica } \\
\text { Biosystems }\end{array}$ & Cat\# DS9665 \\
\hline
\end{tabular}

\section{Experimental Models: Cell Lines}

MCA205 mouse fibrosarcoma cell line

Panel of MCA-derived cancer cell clones

\section{Dr. Oliver}

Kepp, Gustave

Roussy cancer

Campus,

Villejuif

\begin{tabular}{|l|l|}
\hline AT3 mouse mammary carcinoma cell line & Sigma-Aldrich \\
\hline CT26 WT mouse colon carcinoma cell line & ATCC \\
\hline B16.F10 murine melanoma cell line & ATCC \\
\hline MDA-MB-231 human breast carcinoma cell line & ATCC \\
\hline U2OS human bone osteosarcoma cell line & ATCC \\
\hline Experimental Models: Organisms/Strains & $\begin{array}{l}\text { The Jackson } \\
\text { Laboratory }\end{array}$ \\
\hline Mouse: C57BL/6J & The Jackson \\
\hline Mouse : NOD.Cg-Prkdcscid Il2rgtm1Wjl/SzJ (NSG) &
\end{tabular}

Cat\# SCC178, RRID:CVCL_VR89

Cat\#SCC173;
RRID:CVCL_VR90

Cat\# CRL-2638,

RRID:CVCL_7256

Cat\# CRL-6475,

RRID:CVCL_0159

Cat\# HTB-26,

RRID:CVCL_0062

Cat\# HTB-96,

RRID:CVCL_0042

Cat\# 000664; RRID:IMSR_JAX:000664

Cat\# 005557; 


\begin{tabular}{|c|c|c|}
\hline & Laboratory & RRID:IMSR_JAX:005557 \\
\hline Mouse: C57BL/6-Tg(TcraTcrb) 1100Mjb/Crl OT1 & Charles River & Strain Code 642 \\
\hline \multicolumn{3}{|l|}{ Software and Algorithms } \\
\hline Flowjo v10.0.7 & Flowjo, LLC & RRID: SCR_008520 \\
\hline Illustrator CC 2015 & $\begin{array}{l}\text { Adobe Systems } \\
\text { Inc. }\end{array}$ & RRID: SCR_010279 \\
\hline Photoshop CC 2015 & $\begin{array}{l}\text { Adobe Systems } \\
\text { Inc. }\end{array}$ & RRID: SCR_014199 \\
\hline ImageJ & $\begin{array}{l}\text { National } \\
\text { Institute of } \\
\text { Health }\end{array}$ & $\begin{array}{l}\text { http://rsb.info.nih.gov/ij/;_RRID: } \\
\text { SCR_003070 }\end{array}$ \\
\hline Bio-Plex Manager Software version 6.1 & Bio-Rad & RRID:SCR_014330 \\
\hline BWA algorithm & & RRID:SCR_010910 \\
\hline STAR alignment - DESeq2 software & & RRID:SCR_015687 \\
\hline “R" software & $\begin{array}{l}\text { R Foundation } \\
\text { for Statistical } \\
\text { Computing }\end{array}$ & http://www.R-project.org/ \\
\hline HOMER software & & RRID:SCR_010881 \\
\hline \multicolumn{3}{|l|}{ BiNGO software } \\
\hline \multicolumn{3}{|l|}{ EnrichmentMap software } \\
\hline GraphPad Prism & $\begin{array}{l}\text { GraphPad } \\
\text { Software }\end{array}$ & \\
\hline Microsoft Excel & $\begin{array}{l}\text { Microsoft, } \\
\text { Redmond }\end{array}$ & RRID:SCR_016137 \\
\hline SPSS software & SPSS Inc. & \\
\hline \multicolumn{3}{|l|}{ Other } \\
\hline Roswell Park Memorial Institute 1640 (RPMI 1640) & EuroClone & Cat\# ECB9006L \\
\hline Dulbecco's Modified Eagle Medium high glucose (DMEM) & EuroClone & Cat\# ECB7501LX60 \\
\hline Fetal bovine serum (FBS) & EuroClone & Cat\# ECS0180L \\
\hline Penicillin G sodium salt and streptomycin sulfate & EuroClone & Cat\# ECB3001D/1 \\
\hline L-glutamine $200 \mathrm{mM}$ & EuroClone & Cat\# ECB3004D \\
\hline Dulbecco’s Modified Eagle Medium (DMEM) & $\begin{array}{l}\text { Thermo } \\
\text { Scientific }\end{array}$ & Cat\# 52100047 \\
\hline Ham's F-12 Nutrient Mix (F-12) & $\begin{array}{l}\text { Thermo } \\
\text { Scientific }\end{array}$ & Cat\# 21700018 \\
\hline Bovine serum albumin (BSA) & $\begin{array}{l}\text { US Biological, } \\
\text { Salem }\end{array}$ & Cat\# A1312 \\
\hline Penicillin-Streptomycin-Amphotericin B (PSF) & Lonza & Cat\# 17-745E \\
\hline Sodium Bicarbonate $7.5 \%$ solution & $\begin{array}{l}\text { Thermo } \\
\text { Scientific }\end{array}$ & Cat\# 25080094 \\
\hline Hepes buffer $1 \mathrm{M}$ & $\begin{array}{l}\text { Thermo } \\
\text { Scientific }\end{array}$ & Cat\# 15630056 \\
\hline L-glutamine $200 \mathrm{mM}$ & $\begin{array}{l}\text { Thermo } \\
\text { Scientific }\end{array}$ & Cat\# 25030024 \\
\hline Human apotransferrin & Sigma-Aldrich & Cat\# T2252 \\
\hline Heparin sodium salt & Sigma-Aldrich & Cat\# H3393 \\
\hline D-(+)-Glucose solution & Sigma-Aldrich & Cat\# G8769 \\
\hline Recombinant human insulin & Sigma-Aldrich & Cat\# 91077C \\
\hline Progesterone & Sigma-Aldrich & Cat\# P8783 \\
\hline Putrescine dihydrochloride & Sigma-Aldrich & Cat\# P5780 \\
\hline Sodium selenite & Sigma-Aldrich & Cat\# S5261 \\
\hline
\end{tabular}




\begin{tabular}{|l|l|l|} 
Recombinant human epidermal growth factor (EGF) & PeproTech Inc. & Cat\# AF-100-15 \\
\hline $\begin{array}{l}\text { Recombinant human fibroblast growth factor basic } \\
\text { (bFGF) }\end{array}$ & PeproTech Inc. & Cat\# AF-100-18B \\
\hline Nicotinamide & Sigma-Aldrich & Cat\# N0636 \\
\hline Trypsin-EDTA w/ Phenol Red & EuroClone & Cat\# ECM0920 \\
\hline Dulbecco's Phosphate-Buffered Saline (D-PBS) & EuroClone & Cat\# ECB4053L \\
\hline Paraformaldehyde & Sigma-Aldrich & Cat\# 158127 \\
\hline MACS columns & Miltenyi Biotec & $\begin{array}{l}\text { Cat\# 130-042-201, Cat\# 130- } \\
042-401\end{array}$ \\
\hline MACS separators & Miltenyi Biotec & $\begin{array}{l}\text { Cat\# 130-042-109, Cat\# 130- } \\
042-302\end{array}$ \\
\hline
\end{tabular}

\section{CONTACT FOR REAGENT AND RESOURCE SHARING}

Further information and requests for resources and reagents should be directed to and will be fulfilled by the Lead Contact, Antonella Sistigu (antonella.sistigu@unicatt.it).

\section{EXPERIMENTAL MODELS AND METHODS DETAILS}

Cell lines and culture conditions. Unless otherwise indicated, plasticware was from Falcon, Corning B.V. Life Sciences (Corning, NY). MCA205 and MCA205-OVA mouse fibrosarcoma cell line, MCA205-derived

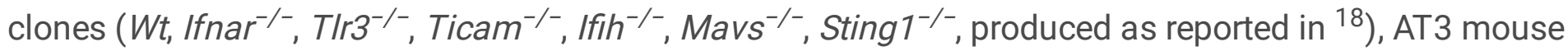
mammary carcinoma and CT26 WT mouse colon carcinoma cell lines were cultured in a Roswell Park Memorial Institute 1640 (RPMI 1640) growth medium supplemented with $10 \%$ (v/v) fetal bovine serum (FBS), $2 \mathrm{mM} \mathrm{L-glutamine,} 100 \mathrm{IU} / \mathrm{mL}$ penicillin $\mathrm{G}$ sodium salt and $100 \mu \mathrm{g} / \mathrm{mL}$ streptomycin sulfate. B16.F10 murine melanoma cells, MDA-MB-231 human breast carcinoma cells and U2OS human bone osteosarcoma cells were cultured in Dulbecco's Modified Eagle Medium high glucose (DMEM) supplemented as above. All cell lines were maintained in standard culture conditions $\left(37^{\circ} \mathrm{C}, 5 \% \mathrm{CO}_{2}\right)$.

For testing the potential of induced cancer stem cells (CSCs) to growth under standard culture conditions already established for colorectal $\operatorname{CSCs}^{60}$, we used the CSC medium described in ${ }^{60}$. In more details DMEM/F12-based culture medium was supplemented with $4 \mathrm{mg} / \mathrm{mL}$ bovine serum albumin (BSA), $1 \mathrm{X}$ Penicillin-Streptomycin-Amphotericin B (PSF), 0.13\% NaHCO3, 6 mM Hepes, 2 mM L-glutamine, 0.1 $\mathrm{mg} / \mathrm{mL}$ apotransferrin, 0.4 units heparin sodium salt, $1.1 \%$ glucose, $25 \mu \mathrm{g} / \mathrm{mL}$ insulin, $6.3 \mathrm{ng} / \mathrm{mL}$ progesterone, $9.7 \mu \mathrm{g} / \mathrm{mL}$ putrescine dihydrochloride, and $5.2 \mathrm{ng} / \mathrm{mL}$ sodium selenite and supplemented with $20 \mathrm{ng} / \mathrm{mL}$ human epidermal growth factor (EGF), $10 \mathrm{ng} / \mathrm{mL}$ human basic fibroblast growth factor (bFGF), and $10 \mathrm{mM}$ nicotinamide. CSCs were passaged once/twice a week at dilution 1:2 by mechanical dissociation, through a micropipette, and incubated in standard culture conditions in ultra-low attachment tissue culture flasks.

Cytofluorometric analysis and cell sorting. To assess the expression of specific surface markers on putative-induced CSCs, $1 \times 10^{5}$ murine and human tumor cells were cultured in 6-well plates in $2 \mathrm{~mL}$ of growth medium and treated $72 \mathrm{~h}$ with purified mouse IFN- $\alpha / \beta$ or recombinant human Roferon- $\mathrm{A}^{\circledR}(6000$ $\mathrm{U} / \mathrm{mL})$ or with DOX $(25 \mu \mathrm{M})$ or OXP $(300 \mu \mathrm{M})$ alone or in combination with TCP $(10 \mu \mathrm{M})$ for $48 \mathrm{~h}$. Cells 
were then collected, washed in Dulbecco's Phosphate-Buffered Saline (D-PBS) and stained with fluorescently labeled mAbs directed against human/murine CD44, CD133 and/or CD24, or with purifiedCD44v6 mAb, at optimal mAb concentrations (dilution 1:20, as previously determined by titration), in a cold D-PBS solution containing 1\% FBS (D-PBS-FBS 1\%). Samples were incubated in the dark on ice for $30 \mathrm{~min}$ and then washed twice with cold D-PBS-FBS $1 \%$ solution. Thereafter, cells stained with CD44v6 $m A b$, were co-stained with the appropriate Alexa Fluor ${ }^{\circledR} 488$ secondary Ab (diluted at 1:500 in D-PBS-FBS $1 \%$ ) on ice for $30 \mathrm{~min}$. Cells were washed twice before the addition of $150 \mu \mathrm{L}$ growth medium supplemented with $1 \mu \mathrm{g} / \mathrm{mL}$ DAPI. Sample acquisitions were performed by means of a BD FACSCanto ${ }^{\mathrm{TM}}$ II (BD Biosciences), MACSQuant ${ }^{\circledR}$ VYB Analyzer 10 (Miltenyi Biotec), or CytoFLEX (Beckman Counter) cytofluorometer, while data were analyzed by using the FlowJo software v10.0.7. By using the same procedure of costaining, specific CSC-like subsets (named ICD-CSCs or IFN-CSCs) were isolated by fluorescence activated cell sorting (FACS) by means of a BD FACSAria ${ }^{\mathrm{TM}}$ cytometer (BD Biosciences) and further characterized by in vitro and in vivo assays.

Other types of cell subsets were also sorted from MCA205 cells first treated with $2.5 \mu \mathrm{M} \mathrm{DOX}$ for $48 \mathrm{~h}$, and then FACS-isolated based on their low or high positivity for red fluorescence, named respectively DOX ${ }^{+l o w}$ and $\mathrm{DOX}^{\text {thigh }}$ cells. For the assessment by flow cytometry of the expression of immune checkpoint molecules, FACS-isolated ICD-CSCs from AT3 and MCA205 cells were stained at $4^{\circ} \mathrm{C}$ for $30 \mathrm{~min}$ in the dark with the following murine fluorochrome-conjugated mAbs directed against: PD-L1 (diluted at 1:100); PDL2CD1LG (diluted at 1:100), LGALS9 (diluted at 1:20), CEACAM1 (diluted at 1:100) and H2-K1 (diluted at 1:50). DAPI was used to distinguish live and dead cells, and analysis of the expression of immune checkpoint molecules was made only in live cells. Samples were then analyzed by means of a MACSQuant ${ }^{\circledR}$ VYB cytofluorometer.

To evaluate how free nucleic acids contribute to the acquisition of CSC traits, $3 \times 10^{5}$ murine tumor cells were cultured in 6-well plates ( $2 \mathrm{~mL}$ of medium/well) and treated with $300 \mu \mathrm{M}$ OXP for $24 \mathrm{~h}$ ("donor" cells). Thereafter, "donor" cells were collected, washed from OXP and incubated at $37^{\circ} \mathrm{C}$ for up to $4 \mathrm{~h}$ in 1,5 $\mathrm{mL}$-eppendorf microtubes containing growth medium, supplemented or not, with $200 \mathrm{IU} / \mathrm{mL}$ BNZase, 10 $\mathrm{IU} / \mathrm{mL}$ RNase A, $10 \mathrm{IU} / \mathrm{mL}$ RNase H or $100 \mathrm{IU} / \mathrm{mL}$ DNase. Next, such "donor cells" were cocultured with untreated live cells ("receiving" cells) for $24 \mathrm{~h}$ in the presence or not of the indicated nucleases before cytofluorometric-mediated assessment of CSC surface markers on "receiving" cells.

Side-population (SP) assay. The detection of CSCs based on the Hoechst 33342 dye efflux [via the ATP binding cassette $(A B C)$ transporter proteins] was performed as previously reported ${ }^{61,62}$. Briefly, $1 \times 10^{5}$ murine tumor cells were cultured in 6-well plates ( $2 \mathrm{~mL}$ of medium/well) and treated with $6000 \mathrm{U} / \mathrm{mL} \mathrm{IFN}$ $\alpha / \beta$ for $72 \mathrm{~h}, 30 \mu \mathrm{M}$ OXP or $2.5 \mu \mathrm{M}$ DOX for $48 \mathrm{~h}$. Cells were then collected, washed and incubated in prewarmed growth medium in the presence or not of $100 \mu \mathrm{M}$ VRP for $30 \mathrm{~min}$ at $37^{\circ} \mathrm{C}$. Five $\mu \mathrm{g} / \mathrm{mL}$ Hoescht 33342 was added to cell suspension for $90 \mathrm{~min}$ at $37^{\circ} \mathrm{C}$ in the dark. Data acquisition was performed using a MACSQuant ${ }^{\circledR}$ VYB cytometer. SP was identified as the distinct Hoechst $33342^{-}$cell population (450-50 $\mathrm{nm}$ band-pass filter channel) within $\mathrm{Pl}^{-}$cells. Data were analyzed by using FlowJo software v10.0.7. 
Quantitative (q)RT-PCR. Total RNA extraction and genomic DNA removal were performed with the RNeasy Plus Mini Kit following manufacturer's instructions. Total RNA (30 ng from each sample) was reverse transcribed and amplified using GoTaq ${ }^{\circledR}$ Probe 1-Step RT-qPCR System in the presence of specific primers and probes for murine genes, following manufacturer's instructions. The following primers were used : Klf4 (Mm00516104_m1), Myc (Mm00487804_m1), Pou5f1 (Mm03053917_g1), Sox2 (Mm03053810_s1), Nanog (Mm02019550_s1), Hes1 (Mm01342805_m1), Nes (Mm00450205_m1), Twist1 (Mm00442036_m1,), Snai1 (Mm00441533_g1), Cdh2 (Mm01162497_m1), Vim (Mm00619195_g1), Fn1 (Mm01256744_m1), Lgals9 (Mm00495295_m1), Pdcd1/g2 (Mm00451734_m1) and Pdl1 (fw: GCATTATATTCACAGCCTGC, rw: CCCTTCAAAAGCTGGTCCTT). QRT-PCR was analyzed on a StepOnePlus Real-Time PCR System. qRT-PCR data were invariably normalized to the expression levels of the housekeeping gene Ppia (Mm00620857_s1).

Semiquantitative RT-PCR. Total RNA extraction and genomic DNA removal were performed as above. RTPCR reaction was carried out, as indicated before in the qRT-PCR section, in the presence of the same specific primers and probes used for qRT-PCR. Amplified products were resolved by electrophoresis in $2 \%$ agarose gel and visualized by using SYBR Safe DNA gel staining.

Clonogenic assay. For assessing the clonogenicity of IFN-induced CSCs (referred to as IFN-CSCs), $1 \times 10^{3}$ IFN- $\alpha / \beta$ pre-treated cancer cells were seeded in 24 -well plates between two layers consisting of $0.4 \%$ agarose for a final volume of $500 \mu \mathrm{L}$ of growth medium supplemented as in ${ }^{60}$ (CSC medium). Cells were incubated under standard culture conditions for up to 15 days. Colonies were then fixed/stained with $0.02 \%$ crystal violet (diluted in $20 \%$ methanol) and counted under an inverted microscope. Some of these spheres, prior to fixation, were recovered, cultured in ultra-low attachment flasks in CSC medium and analyzed for their morphology and transcriptional profiles.

Multidrug resistance assay. To determine CSC resistance to conventional chemotherapeutics, $5 \times 10^{3}$ parental and IFN-CSC MCA205 cells were seeded in 96-well plates (90 $\mathrm{LL}$ of medium/well) and either left untreated ("0") or treated with growing doses of OXP (3-30-300 $\mu \mathrm{M})$, DOX (0.25-2.5-25 $\mu \mathrm{M})$ and MTX (0.04-0.4-4 $\mu \mathrm{M})$ for $72 \mathrm{~h}$. Cell viability/proliferation was determined by luminescence counting of ATP levels in each well using the CellTiter-Glo ${ }^{\circledR}$ Luminescent Cell Viability Assay and a multimode reader (DTX-880; Beckman Coulter) according to manufacturer's instructions.

T cell proliferation and cancer cell killing assays. MCA205-OVA were UV irradiated as in ${ }^{63}$ and co-cultured with BM-derived DCs (differentiated as in ${ }^{64}$ ) at a 2:1 ratio for $24 \mathrm{~h}$. DCs were then cultured at a 5:1 ratio with splenic purified CD8 ${ }^{+}$OT-1 cells for $72 \mathrm{~h}$. Cross-primed CD $8^{+}$OT- 1 cells were then labelled with $1 \mu \mathrm{M}$ CFSE dye (Sigma Aldrich) for $10 \mathrm{~min}$ at $37^{\circ} \mathrm{C}$, and re-stimulated with live parental or CD44L MCA205-OVA cells at 1:5 ratio. Three days later, cells were recovered and analyzed by flow cytometry (CytoFLEX, Beckman Coulter) for CFSE levels on live gated CD8 ${ }^{+}$cells and PI levels on CD45 cells. Data were analyzed by using FlowJo software v10.0.7. 
Microfluidic co-culture system for tumor-immune interactions. Microfluidic devices were fabricated in polydimethylsiloxane following well-established replica molding procedures ${ }^{65}$. Prior to cell loading, each device was sterilized under UV light in a laminar flow hood for 30min and then filled with RPMI 1640 growth medium and incubated for $1 \mathrm{~h}$ at $37^{\circ} \mathrm{C}$ to equilibrate the system. To follow chemical and physical contacts between tumor cells and immune cells, parental AT3 cells, or their ICD-induced CSC counterparts (referred to as ICD-CSCs), were co-cultured with histocompatible H-2Kb splenocytes from C57BL/6J mice. Cells were loaded into the reservoirs of microfluidic devices as follows: the chambers were filled with 200 $\mu$ l RPMI 1640 growth medium containing on one side $2 \times 10^{6}$ mouse splenocytes and on the other $5 \times 10^{4}$ tumor cells. Through an array of narrow micro-channels, immune cells can migrate toward tumor cells. Time-lapse recordings were carried out over a period of $72 \mathrm{~h}$ by means of a Juli Smart microscope (Bulldog Bio Inc, Porthsmouth, NH) placed inside the incubator for all the duration of the recordings. The microscope generated 1 microphotograph every $2 \mathrm{~min}$, for a total of 720 images per day. Tracking analysis of timelapse microphotographs was performed by using the Manual Tracking plug-in of the ImageJ v1.5 software.

Microfluidic devices based on competition. To concomitantly confront the immune cells with different tumor cell populations, $2 \times 10^{4}$ parental AT3 cells and their ICD-CSC counterparts were resuspended on ice in $3 \mu \mathrm{L}$ Matrigel $(2 \mathrm{mg} / \mathrm{mL})$, loaded in two opposite lateral chambers and mantained at $37^{\circ} \mathrm{C}$ for $30 \mathrm{~min}$ to allow gel solidification. Then, $1 \times 10^{6}$ histocompatible splenocytes, labeled with live-compatible PKH26 Red Fluorescent Cell Linker following manufacturer's instructions, were loaded in the central chamber in $10 \mu$ l of RPMI 1640 growth medium 66,67 . Phase-contrast, visible and fluorescence microphotographs were generated by using an EVOS-FL fluorescence microscope (Life Technologies Thermo Scientific). Fluorescence analysis and image overlays were performed by using ImageJ v1.5 software.

Extracellular vesicle (EV) isolation and uptake. To assess the involvement of EV-carried nucleic acids in the induction of CSCs, $3 \times 10^{5}$ MCA205 cells were seeded in 6-well plates ( $2 \mathrm{~mL}$ of medium/well), treated with $300 \mu \mathrm{M}$ OXP for $4 \mathrm{~h}$ ("donor" cells) and then washed. Purification of EVs from cell culture supernatant was performed using exoEasy Maxi Kit following manufacturer's instructions. Isolated EVs were put on "receiving cells" for $24 \mathrm{~h}$ in presence or not of the endocytosis inhibitor cytochalasin D (0.5 $\mu \mathrm{M})$. "Receiving” cells were then analyzed by cytofluorometry and qRT-PCR. For uptake analysis, isolated EVs were labeled with the PKH26 Red Fluorescent Cell Linker, washed with Exosome Spin Columns (Cat\# 4484449, Invitrogen - Thermo Scientific) and co-cultured for $4 \mathrm{~h}$ with "receiving" cells either at $37^{\circ} \mathrm{C}$ or $4^{\circ} \mathrm{C}$. Thereafter, cells were washed, fixed in $4 \%(\mathrm{w} / \mathrm{v})$ paraformaldehyde in D-PBS, and analyzed by flow cytometry and EVOS-FL fluorescence microscope.

Luminex assay. To evaluate the basal differences in chemokine patterns, parental MCA205 cells, parental AT3 cells and their respective ICD-CSC counterparts were seeded in 24-well plates ( $1 \mathrm{~mL} \mathrm{medium} /$ well) and grown for $48 \mathrm{~h}$. Supernatants were collected on ice, centrifuged and immediately freezed at $-80^{\circ} \mathrm{C}$. Chemokines were measured by a xMAP multiplex technology using a Mouse Magnetic Luminex assay 
multiplex panel including the following molecules: CCL2/MCP-1/JE; CCL3/MIP-1a; CCL4/MIP-1 $\beta$; CCL5/RANTES; CCL8/MCP-2; CCL11/Eotaxin; CCL12/MCP-5; CCL22/MDC; CXCL1/KC; CXCL2/MIP-2; CXCL10/IP-10; CXCL12/SDF-1a. The analysis was performed by using $50 \mu \mathrm{L}$ of 2 -fold diluted samples. The quantification was carried out on a Bio-Plex ${ }^{\circledR} 200$ System (Bio-Rad, Hercules, CA) equipped with a magnetic workstation and a Bio-Plex Manager Software version 6.1 and results were expressed as $\mathrm{pg} / \mathrm{mL}$. The level of each detected molecule was normalized to the total cell number.

Assay for transposase-accessible chromatin using sequencing (ATAC-seq). To investigate chromatin accessibility dynamics, $1 \times 10^{5}$ parental MCA205 cells and their IFN-CSC counterparts were treated with DNase I at $37^{\circ} \mathrm{C}$ for $30 \mathrm{~min}$. Cells were then washed and cryopreserved in culture medium containing FBS and $5 \%$ DMSO in 1,5 mL eppendorf microtubes. Cryopreserved cells were sent to Epigenetics Services Active Motif, Inc. (Carlsband, CA) to perform the ATAC-seq assay. Cells were thawed in a $37^{\circ} \mathrm{C}$ water bath, pelleted, washed with cold PBS, and tagmented as previously described ${ }^{68}$, with some modifications based on ${ }^{69}$. Briefly, cell pellets were resuspended in lysis buffer, pelleted, and tagmented using the enzyme and buffer provided in the Nextera Library Prep Kit. Tagmented DNA was then purified using the MinElute PCR purification kit, amplified with 10 cycles of PCR, and purified using Agencourt AMPure SPRI beads. Resulting material was quantified using the KAPA Library Quantification Kit for Illumina platforms, and sequenced with PE42 sequencing on the NextSeq 500 sequencer (Illumina). Analysis of ATAC-seq data was performed as follows. Reads were aligned to the mouse genome (mm10) using the BWA algorithm. Duplicate reads were removed, only reads mapping as matched pairs and only uniquely mapped reads (mapping quality $\geq 1$ ) were used for further analysis. Alignments were extended in silico at their 3'-ends to a length of $200 \mathrm{bp}$ and assigned to 32-nt bins along the genome. The resulting histograms (genomic "signal maps") were stored in bigWig files. Peaks were identified using the MACS 2.1.0 algorithm at a cutoff of $p$-value 1e-7, without control file, and with the -nomodel option. Peaks that were on the ENCODE blacklist of known false ChIP-Seq peaks were removed. Signal maps and peak locations were used as input data to Active Motifs proprietary analysis program, which creates Excel tables containing detailed information on sample comparison, peak metrics, peak locations and gene annotations. A peak re-calling strategy was used to reduce false positives as previously done in 70 .

RNA-seq. To determine the overall transcriptional profile, $2.5 \times 10^{5}$ parental MCA205 cells and their IFNCSC counterparts were harvested, washed and cryopreserved in 1,5 mL eppendorf microtubes. As above, RNA-seq analysis was performed by Epigenetics Services Active Motif, Inc. Total RNA was isolated from cells by using the RNeasy Mini Kit. For each sample, $2 \mu \mathrm{g}$ of total RNA was then used in Illumina's TruSeq Stranded mRNA Library kit. Libraries were sequenced on Illumina NextSeq 500 as paired-end 42-nt reads. Sequence reads were analyzed with the STAR alignment - DESeq2 software pipeline described in the Data Explanation document.

Transcription factor motif discovery and network analysis. Motif enrichment analysis was performed using the HOMER software comparing the motifs enriched in the target set (i.e., the loci obtained from ATAC-Seq analysis) with those of reference (i.e., randomly selected background sequences). Only motif 
ratios $\geq 2$ with $P$-value (Benjamini-Hochberg correction) $\leq 0.05$ were considered biologically and statistically significant. The functional enrichment analysis was performed by using the clusterProfiler package as in ${ }^{71}$. Network visualizations were made by using the enrichPlot package.

Animals. Mice were maintained in specific pathogen-free conditions in a temperature-controlled environment with $12 \mathrm{~h}$ light $-12 \mathrm{~h}$ dark cycles and received food and water ad libitum. All the in vivo experimentations were in compliance with the EU Directive 63/2010 and included in an experimental protocol approved by the Institutional Animal Experimentation Committee (858/2015-PR). Six-to-7 weekold female C57BI/6J, and NOD SCID gamma (NSG) mice were purchased from Jackson Laboratory, C57BL/6-Tg(TcraTcrb)1100Mjb/J OT1 mice were from Charles River, housed in the animal facility at the Istituto Superiore di Sanità and employed after an acclimatization period of 7 days. All experiments were randomized and blinded and sample sizes were calculated to detect a statistically significant effect.

Tumor models, vaccination and chemotherapy. To assess cancer cell tumorigenic capacity, $1 \times 10^{2} ; 1 \mathrm{x}$ $10^{3} ; 1 \times 10^{4} ; 1 \times 10^{5}$ parental MCA205 and and their IFN-CSC counterparts were subcutaneously inoculated into the flank of C57BI/6J and NSG mice. Tumor surface (longest dimension x perpendicular dimension) was routinely monitored using a common caliper. For vaccination experiments, $1 \times 10^{5}$ parental MCA205 cells were subcutaneously inoculated in mice which rejected the first injection, and tumor growth was monitored weekly. The absence of tumors was considered an indication of efficient antitumor vaccination.

To in vivo uncover the ability of immunogenic chemotherapy to induce CSC appearance and to test IFNCSC therapy response, $1 \times 10^{6}$ parental or IFN-CSC MCA205 were subcutaneously inoculated into the flank of $\mathrm{C} 57 \mathrm{Bl} / 6 \mathrm{~J}$ mice and tumor growth was weekly monitored (as above described). When the tumor surface reached $35-45 \mathrm{~mm}^{2}$ mice were randomized to control and treatment groups (10 mice/group) and injected with either CDDP $(2.5 \mathrm{mg} / \mathrm{kg})$ or DOX $(2.9 \mathrm{mg} / \mathrm{kg})$, both intratumorally in $50 \mu$ l of D-PBS, TCP $(5$ $\mathrm{mg} / \mathrm{kg}$ ) intraperitoneally every 3 days alone, a combination of DOX+TCP, or $50 \mu \mathrm{l}$ of D-PBS intratumorally. All experiments contained 5 to 10 mice per group and were run at least 2 times, yielding similar results. All data were analyzed by using GraphPad Prism.

Tumor dissection, flow cytometry and sorting. Tumors from mice either treated with CDDP, DOX, D-PBS, TCP, DOX+TCP were carefully removed 15 days after treatment. Tumor burdens were cut into small pieces with scissors within digesting buffer (400 U/ml Collagenase A and $200 \mathrm{U} / \mathrm{ml}$ DNase I in RPMI 1640) and incubated for $30 \mathrm{~min}$ at $37^{\circ} \mathrm{C}$. Single cell suspensions obtained by grinding the digested tissue and filtering them through a 70- $\mu \mathrm{m}$ cell strainer were then purified based on CD45 expression, by using mouse CD45 MicroBeads, MACS columns and separators (used following manufacter's recommandations).

After washing with D-PBS, CD45 ${ }^{+}$cells, uncluding tumor infiltrating lymphocytes (TILs), were resuspended at $1 \times 10^{7}$ cells $/ \mathrm{mL}$ and stained at $4^{\circ} \mathrm{C}$ for $30 \mathrm{~min}$ in the dark with the following murinespecific fluorochrome-conjugated mAbs directed against: CD45 (diluted at 1:25); CD8a (diluted at 1:150); and TIM-3 (diluted at 1:100). Similarly, the CD $45^{\circ}$ cellular fraction (including tumor cells) was stained as 
follows: CD45, CD133, CD44, CD24 and Nanog (diluted at 1:5). DAPI was used to distinguish live and dead cells and only live cells were included in the analysis. Samples were then analysed by means of a MACSQuant ${ }^{\circledR}$ VYB or a CytoFLEX cytofluorometer.

In vivo invasiveness assay. For invasiveness studies, $2 \times 10^{5}$ parental MCA205 cells and their ICD-CSCs counterparts were intravenously injected into the tail of C57BI/6J mice. Fiftheen days post injection, lungs were explanted and macrometastases were counted. Images of lung metastases were captured by using a ZEISS STEMI 305 Stereo microscope (Carl Zeiss, Oberkochen, Germany). All experiments contained 5 to 10 mice per group and were run at least 2 times, yielding similar results. All data were analyzed by using GraphPad Prism.

Immunohistochemistry (IHC). Three-micrometer sections of formalin-fixed paraffin embedded breast cancer (BC) biopsies and autologous surgery tissues were cut on SuperFrost Plus slides (Menzel-Gläser, Braunschweig, Germany). Immunoreactions against CD45 and MX1 were revealed by Bond Polymer Refine Detection in an automated autostainer (Bond III, Leica Biosystems, Wetzlar, Germany) using the following antibodies: anti-mouse CD45 mAb (clone 2B11, dilution of 1:100, pH 6) and anti-rabbit MX1 polyclonal $\mathrm{Ab}$ (dilution of 1:100, $\mathrm{pH}$ 6). Diaminobenzidine was used as chromogenic substrate. The immunohistochemical assessment of CD44 and CD24 was performed with ChromoPlex TM1 Dual Detection in an automated autostainer by using an anti-rabbit CD44 mAb (clone SP37, dilution of 1:150, pH 6) and anti-mouse CD24 mAb (clone SN3, dilution of 1:500, pH 8). Diaminobenzidine and Fast Red were used as chromogenic substrates. Immunoreactions were scored independently by 2 investigators ( $C E$ and $A D B$ ). Blinded to treatment outcomes and discordant cases were reviewed for the final assessment.

Patients included in neoadjuvant chemotherapy studies. For IHC retrospective analyses 20 patients, with histologically confirmed BC by the Pathology Unit at the Regina Elena National Cancer Institute (Rome), were included. All the patients underwent biopsies and received anthracycline-based neoadjuvant chemotherapy. For metagene correlation analyses, the publicly available patient cohorts (accession codes GSE6861, GSE20271, GSE25065, GSE16446, GSE41998 and GSE32646) reported in 18, 37 were selected. In these studies gene expression analyses were performed on tumor biopsies obtained at diagnosis.

Statistical Analysis. Unless otherwise specified, all experiments were performed in triplicate parallel instances and independently repeated at least three times. Shapiro-Wilk Normality Test on SPSS software Version_21 was used to assess the presence or not of parametric distributions. According to these results, statistical significance of data from most in vitro and in vivo studies was evaluated by: ( $I$ ) unpaired $t$ test with Welch's correction; (ii) Mann-Whitney non-parametric test, (iii) ordinary one- or two-way ANOVA with Bonferroni's correction. The rate of migration of splenocytes into microfluidic devices was analyzed by two-way repeated measures ANOVA. For in vivo studies, the Log-rank (Mantel-Cox) test was applied to compare tumor growth curves. For IHC analyses, the Spearman's correlation coefficient was calculated to 
assess the correlation between MX1 and CD44, CD24 markers. $P$ values $<0.05$ were considered statistically significant.

\section{References}

1. Gottesman, M.M. Mechanisms of cancer drug resistance. Annu Rev Med 53, 615-627 (2002).

2. Russo, M. et al. Adaptive mutability of colorectal cancers in response to targeted therapies. Science (2019).

3. Gatenby, R.A. \& Brown, J.S. Integrating evolutionary dynamics into cancer therapy. Nature reviews. Clinical oncology 17, 675-686 (2020).

4. Gilbert, L.A. \& Hemann, M.T. DNA damage-mediated induction of a chemoresistant niche. Cell 143, 355-366 (2010).

5. Lagadec, C., Vlashi, E., Della Donna, L., Dekmezian, C. \& Pajonk, F. Radiation-induced reprogramming of breast cancer cells. Stem Cells 30, 833-844 (2012).

6. Dahan, P. et al. lonizing radiations sustain glioblastoma cell dedifferentiation to a stem-like phenotype through survivin: possible involvement in radioresistance. Cell Death Dis 5, e1543 (2014).

7. Lu, H. et al. Chemotherapy-Induced Ca(2+) Release Stimulates Breast Cancer Stem Cell Enrichment. Cell Rep 18, 1946-1957 (2017).

8. Dean, M., Fojo, T. \& Bates, S. Tumour stem cells and drug resistance. Nature reviews. Cancer 5, 275284 (2005).

9. Batlle, E. \& Clevers, H. Cancer stem cells revisited. Nature medicine 23, 1124-1134 (2017).

10. Vitale, I., Shema, E., Loi, S. \& Galluzzi, L. Intratumoral heterogeneity in cancer progression and response to immunotherapy. Nature medicine 27, 212-224 (2021).

11. Cao, J. \& Yan, Q. Cancer Epigenetics, Tumor Immunity, and Immunotherapy. Trends in cancer 6, 580$592(2020)$.

12. Wainwright, E.N. \& Scaffidi, P. Epigenetics and Cancer Stem Cells: Unleashing, Hijacking, and Restricting Cellular Plasticity. Trends in cancer 3, 372-386 (2017).

13. Cheng, Y. et al. Targeting epigenetic regulators for cancer therapy: mechanisms and advances in clinical trials. Signal transduction and targeted therapy 4, 62 (2019).

14. Dawson, M.A. \& Kouzarides, T. Cancer epigenetics: from mechanism to therapy. Cell 150, 12-27 (2012).

15. Topper, M.J., Vaz, M., Marrone, K.A., Brahmer, J.R. \& Baylin, S.B. The emerging role of epigenetic therapeutics in immuno-oncology. Nature reviews. Clinical oncology 17, 75-90 (2020).

16. Villanueva, L., Alvarez-Errico, D. \& Esteller, M. The Contribution of Epigenetics to Cancer Immunotherapy. Trends in immunology 41, 676-691 (2020).

17. Schiavoni, G. et al. Cyclophosphamide synergizes with type I interferons through systemic dendritic cell reactivation and induction of immunogenic tumor apoptosis. Cancer Res 71, 768-778 (2011). 
18. Sistigu, A. et al. Cancer cell-autonomous contribution of type I interferon signaling to the efficacy of chemotherapy. Nature medicine 20, 1301-1309 (2014).

19. Galluzzi, L., Buque, A., Kepp, O., Zitvogel, L. \& Kroemer, G. Immunogenic cell death in cancer and infectious disease. Nat Rev Immunol 17, 97-111 (2017).

20. Galluzzi, L. et al. Molecular mechanisms of cell death: recommendations of the Nomenclature Committee on Cell Death 2018. Cell Death Differ 25, 486-541 (2018).

21. Musella, M., Manic, G., De Maria, R., Vitale, I. \& Sistigu, A. Type-l-interferons in infection and cancer: Unanticipated dynamics with therapeutic implications. Oncoimmunology 6, e1314424 (2017).

22. Hugo, W. et al. Genomic and Transcriptomic Features of Response to Anti-PD-1 Therapy in Metastatic Melanoma. Cell 165, 35-44 (2016).

23. Benci, J.L. et al. Opposing Functions of Interferon Coordinate Adaptive and Innate Immune Responses to Cancer Immune Checkpoint Blockade. Cell 178, 933-948 e914 (2019).

24. Dunn, G.P. et al. A critical function for type I interferons in cancer immunoediting. Nature immunology 6, 722-729 (2005).

25. Lee, J. et al. Activation of innate immunity is required for efficient nuclear reprogramming. Cell 151, 547-558 (2012).

26. Fang, R. et al. Human LSD2/KDM1b/AOF1 regulates gene transcription by modulating intragenic H3K4me2 methylation. Molecular cel/39, 222-233 (2010).

27. Al-Hajj, M., Wicha, M.S., Benito-Hernandez, A., Morrison, S.J. \& Clarke, M.F. Prospective identification of tumorigenic breast cancer cells. Proc Natl Acad Sci U S A 100, 3983-3988 (2003).

28. Bocci, F. et al. Toward understanding cancer stem cell heterogeneity in the tumor microenvironment. Proc Natl Acad Sci U S A 116, 148-157 (2019).

29. Marine, J.C., Dawson, S.J. \& Dawson, M.A. Non-genetic mechanisms of therapeutic resistance in cancer. Nature reviews. Cancer 20, 743-756 (2020).

30. Balkwill, F. Cancer and the chemokine network. Nature reviews. Cancer 4, 540-550 (2004).

31. Acharyya, S. et al. A CXCL1 paracrine network links cancer chemoresistance and metastasis. Cell 150, 165-178 (2012).

32. Wiedemann, G.M. et al. Cancer cell-derived IL-1alpha induces CCL22 and the recruitment of regulatory T cells. Oncoimmunology 5, e1175794 (2016).

33. Vacchelli, E. et al. Chemotherapy-induced antitumor immunity requires formyl peptide receptor 1 . Science 350, 972-978 (2015).

34. Dixon, G. et al. QSER1 protects DNA methylation valleys from de novo methylation. Science $\mathbf{3 7 2}$ (2021).

35. Lu, H. et al. Chemotherapy-induced S100A10 recruits KDM6A to facilitate OCT4-mediated breast cancer stemness. The Journal of clinical investigation 130, 4607-4623 (2020).

36. Zhang, W. et al. Targeting KDM4A epigenetically activates tumor-cell-intrinsic immunity by inducing DNA replication stress. Molecular cell (2021). 
37. Hollern, D.P. et al. B Cells and T Follicular Helper Cells Mediate Response to Checkpoint Inhibitors in High Mutation Burden Mouse Models of Breast Cancer. Cell 179, 1191-1206 e1121 (2019).

38. Barrat, F.J., Crow, M.K. \& Ivashkiv, L.B. Interferon target-gene expression and epigenomic signatures in health and disease. Nature immunology 20, 1574-1583 (2019).

39. Park, S.H. et al. Type I interferons and the cytokine TNF cooperatively reprogram the macrophage epigenome to promote inflammatory activation. Nature immunology 18, 1104-1116 (2017).

40. Zitvogel, L., Galluzzi, L., Kepp, O., Smyth, M.J. \& Kroemer, G. Type I interferons in anticancer immunity. Nat Rev Immunol 15, 405-414 (2015).

41. Bracci, L., Sistigu, A., Proietti, E. \& Moschella, F. The added value of type I interferons to cytotoxic treatments of cancer. Cytokine Growth Factor Rev 36, 89-97 (2017).

42. Doherty, M.R. et al. Interferon-beta represses cancer stem cell properties in triple-negative breast cancer. Proc Natl Acad Sci U S A 114, 13792-13797 (2017).

43. Castiello, L. et al. Disruption of IFN-I Signaling Promotes HER2/Neu Tumor Progression and Breast Cancer Stem Cells. Cancer Immunol Res 6, 658-670 (2018).

44. Zhu, Y. et al. Influence of interferon-alpha on the expression of the cancer stem cell markers in pancreatic carcinoma cells. Exp Cell Res 324, 146-156 (2014).

45. Qadir, A.S. et al. CD95/Fas Increases Stemness in Cancer Cells by Inducing a STAT1-Dependent Type I Interferon Response. Cell Rep 18, 2373-2386 (2017).

46. Li, S. et al. Interferon alpha-inducible protein 27 promotes epithelial-mesenchymal transition and induces ovarian tumorigenicity and stemness. J Surg Res 193, 255-264 (2015).

47. Meacham, C.E. \& Morrison, S.J. Tumour heterogeneity and cancer cell plasticity. Nature 501, 328-337 (2013).

48. Turajlic, S., Sottoriva, A., Graham, T. \& Swanton, C. Resolving genetic heterogeneity in cancer. Nat Rev Genet 20, 404-416 (2019).

49. Maccalli, C., Volonte, A., Cimminiello, C. \& Parmiani, G. Immunology of cancer stem cells in solid tumours. A review. Eur J Cancer 50, 649-655 (2014).

50. Miao, Y. et al. Adaptive Immune Resistance Emerges from Tumor-Initiating Stem Cells. Cell 177, 1172-1186 e1114 (2019).

51. Jacquelot, N. et al. Sustained Type I interferon signaling as a mechanism of resistance to PD-1 blockade. Cell Res (2019).

52. Chen, J. et al. Type I IFN protects cancer cells from CD8+ T cell-mediated cytotoxicity after radiation. The Journal of clinical investigation 129, 4224-4238 (2019).

53. Fan, J.B. et al. Type I Interferon Regulates a Coordinated Gene Network to Enhance Cytotoxic T CellMediated Tumor Killing. Cancer Discov (2020).

54. Keklikoglou, I. et al. Chemotherapy elicits pro-metastatic extracellular vesicles in breast cancer models. Nat Cell Biol 21, 190-202 (2019). 
55. Wu, X. et al. Intrinsic Immunity Shapes Viral Resistance of Stem Cells. Cell 172, 423-438 e425 (2018).

56. Sheng, W. et al. LSD1 Ablation Stimulates Anti-tumor Immunity and Enables Checkpoint Blockade. Cell 174, 549-563 e519 (2018).

57. Qin, Y. et al. Inhibition of histone lysine-specific demethylase 1 elicits breast tumor immunity and enhances antitumor efficacy of immune checkpoint blockade. Oncogene 38, 390-405 (2019).

58. Wu, L. et al. KDM5 histone demethylases repress immune response via suppression of STING. PLoS biology 16, e2006134 (2018).

59. Topper, M.J. et al. Epigenetic Therapy Ties MYC Depletion to Reversing Immune Evasion and Treating Lung Cancer. Cell 171, 1284-1300 e1221 (2017).

60. Manic, G. et al. CHK1-targeted therapy to deplete DNA replication-stressed, p53-deficient, hyperdiploid colorectal cancer stem cells. Gut 67, 903-917 (2018).

61. Golebiewska, A., Brons, N.H., Bjerkvig, R. \& Niclou, S.P. Critical appraisal of the side population assay in stem cell and cancer stem cell research. Cell Stem Cell 8, 136-147 (2011).

62. Telford, W.G. Stem cell side population analysis and sorting using DyeCycle violet. Curr Protoc Cytom Chapter 9, Unit9 30 (2010).

63. Lorenzi, S. et al. Type I IFNs control antigen retention and survival of CD8alpha(+) dendritic cells after uptake of tumor apoptotic cells leading to cross-priming. Journal of immunology $186,5142-5150$ (2011).

64. Roney, K. Bone Marrow-Derived Dendritic Cells. Methods in molecular biology 1960, 57-62 (2019).

65. Businaro, L. et al. Cross talk between cancer and immune cells: exploring complex dynamics in a microfluidic environment. Lab Chip 13, 229-239 (2013).

66. Lucarini, V. et al. Combining Type I Interferons and 5-Aza-2'-Deoxycitidine to Improve Anti-Tumor Response against Melanoma. J Invest Dermatol 137, 159-169 (2017).

67. Parlato, S. et al. 3D Microfluidic model for evaluating immunotherapy efficacy by tracking dendritic cell behaviour toward tumor cells. Sci Rep 7, 1093 (2017).

68. Buenrostro, J.D., Giresi, P.G., Zaba, L.C., Chang, H.Y. \& Greenleaf, W.J. Transposition of native chromatin for fast and sensitive epigenomic profiling of open chromatin, DNA-binding proteins and nucleosome position. Nat Methods 10, 1213-1218 (2013).

69. Corces, M.R. et al. An improved ATAC-seq protocol reduces background and enables interrogation of frozen tissues. Nat Methods 14, 959-962 (2017).

70. Li, D. et al. Chromatin Accessibility Dynamics during iPSC Reprogramming. Cell Stem Cell 21, 819833 e816 (2017).

71. Yu, G., Wang, L.G., Han, Y. \& He, Q.Y. clusterProfiler: an R package for comparing biological themes among gene clusters. Omics : a journal of integrative biology 16, 284-287 (2012).

\section{List Of Genes And Proteins}


Actb, actin, beta [Mus musculus]; Aldh/Aldh3a1, aldehyde dehydrogenase family 3, subfamily A1 [Mus musculus]; Aldh5a1, aldhehyde dehydrogenase family 5, subfamily A1 [Mus musculus]; ANXA1, annexin A1 [Mus musculus]; Atf1, activating transcription factor 1 [Mus musculus]; Atf4, activating transcription factor 4 [Mus musculus]; Atp2a3, ATPase, Ca++ transporting, ubiquitous [Mus musculus]; Baiap2, brainspecific angiogenesis inhibitor 1-associated protein 2 [Mus musculus]; Bcl11a, B cell CLL/lymphoma 11A (zinc finger protein) [Mus musculus]; CCL2, chemokine (C-C motif) ligand 2 [Homo sapiens]; CCL22, chemokine (C-C motif) ligand 22 [Mus musculus]; CCL5, chemokine (C-C motif) ligand 5 [Mus musculus]; CD24, CD24 antigen [Mus musculus]; CD24, CD24 molecule [Homo sapiens]; Cd274/Pd11, CD274 antigen [Mus musculus]; CD274/PD-L1, CD274 molecule/programmed death-ligand 1 [Homo sapiens]; CD44, CD44 antigen [Mus musculus ]; CD44, CD44 molecule (Indian blood group) [Homo sapiens]; Cdh1, cadherin 1 [Mus musculus]; Cdh16, cadherin 16 [Mus musculus]; Cdh2, cadherin 2 [Mus musculus]; Cdk2ap1, CDK2 (cyclin-dependent kinase 2)-associated protein 1 [Mus musculus]; CEACAM1/CD66a, carcinoembryonic antigen-related cell adhesion molecule 1 [Mus musculus]; cGAS, cyclic GMP-AMP synthase [Mus musculus]; Csf1r, colony stimulating factor 1 receptor [Mus musculus]; CTCF, CCCTCbinding factor [Mus musculus]; Ctsl, cathepsin L [Mus musculus]; CXCL1, chemokine (C-X-C motif) ligand 1 [Mus musculus]; CXCL10, C-X-C motif chemokine ligand 10 [Homo sapiens]; CXCL2, chemokine (C-X-C motif) ligand 2 [Mus musculus]; DDX58, DEAD (Asp-Glu-Ala-Asp) box polypeptide 58 [Mus Musculus]; Dlg2, discs large MAGUK scaffold protein 2 [Mus musculus]; DLL3, delta like canonical Notch ligand 3 [Homo sapiens]; EBF, early B cell factor [Mus musculus]; Epb4.1/1, erythrocyte membrane protein band 4.1[Mus musculus]; Ets1, E26 avian leukemia oncogene 1, 5' domain [Mus musculus]; Fank, fibronectin type 3 and ankyrin repeat domains 1 [Mus musculus]; Fbxw2, F-box and WD-40 domain protein 2 [Mus musculus]; Fn1, fibronectin [Mus musculus]; FOX, Forkhead box [Mus musculus]; Gapdh, glyceraldheyde3-phosphate dehydrogenase [Mus musculus]; Gata6, GATA binding protein 6 [Mus musculus]; Gpr17, G protein-coupled receptor 17 [Mus musculus]; Gsdmd, gasdermin D [Mus musculus]; $\mathrm{H} 2-\mathrm{K} 1$, histocompatibility 2, K1, K region [Mus musculus]; HAVCR2/CD366, Hepatitis A virus cellular receptor 2 [Mus musculus]; HES1, hes family bHLH transcription factor 1 [Homo sapiens]; Hes1, hes family bHLH transcription factor 1 [Mus musculus]; Hes2, hes family bHLH transcription factor 2 [Mus musculus]; HMGB1, high mobility group box 1 [Mus musculus]; Hnf1b,HNF1 homeobox N [Mus musculus]; IFI27, interferon alpha inducible protein 27 [Homo sapiens]; Ifi27l2a, interferon, alpha-inducible protein 27 like 2A [Mus musculus]; Ifi27l2b, interferon, alpha-inducible protein 27 like 2B [Mus musculus]; Ifih1, interferon induced with helicase $\mathrm{C}$ domain 1 [Mus musculus]; Ifnar1, interferon (alpha and beta) receptor 1 [Mus musculus]; IFNB1, interferon beta 1 [Homo sapiens]; II12b, interleukin 12b [Mus musculus]; II16, interleukin 16 [Mus musculus]; II24, interleukin 24 [Mus musculus]; II27, interleukin 27 [Mus musculus]; IRF, interferon regulatory factor [Mus musculus]; Itga5, integrin alpha 5 (fibronectin receptor alpha) [Mus musculus]; Jun/AP1, jun proto-oncogene [Mus musculus]; Kdm1b, lysine (K)-specific demethylase 1B [Mus musculus]; KDM1B, lysine demethylase 1B [Homo sapiens]; KIf4, Kruppel-like factor 4 (gut) [Mus musculus]; KLF4, Kruppel-like factor 4 [Homo sapiens]; Krt, keratin [Mus musculus]; LGALS9, lectin, galactose binding, soluble 9 [Mus musculus]; LGR5, leucine-rich repeat containing G protein-coupled receptor 5 [Homo sapiens]; MafB, v-maf musculoaponeurotic fibrosarcoma oncogene family, protein B (avian) [Mus musculus]; MafK, v-maf musculoaponeurotic fibrosarcoma oncogene family, protein K

Page 31/46 
(avian) [Mus musculus]; MAVS, mitochondrial antiviral signaling protein [Mus musculus]; Mtor, mechanistic target of rapamycin kinase [Mus musculus]; MX1, MX dynamin like GTPase 1 [Homo sapiens]; Myc myelocytomatosis oncogene [Mus musculus]; MYC, MYC proto-oncogene, bHLH transcription factor [Homo sapiens]; Myct1, myc target 1 [Mus musculus]; NANOG, Nanog homeobox [Homo sapiens]; Nanog, Nanog homeobox [Mus musculus]; Ncam/Ncam1, neural cell adhesion molecule 1 [Mus musculus]; NES, nestin [Homo sapiens]; Nes, nestin [Mus musculus]; NF1, neurofibromin 1 [Mus musculus]; NFAT, Nuclear factor of activated T-cells [Mus musculus]; NFKB1/NFkB-p50, nuclear factor of kappa light polypeptide gene enhancer in B cells 1, p105 [Mus musculus]; NOS2, nitric oxide synthase 2, inducible [Mus musculus]; OASL, 2'-5'-oligoadenylate synthetase like [Homo sapiens]; PAX3, paired box 3 [Mus musculus]; PDCD1LG2/PD-L2, programmed cell death 1 ligand 2 [Homo sapiens]; Pdcd1lg2/PD-L2, programmed cell death 1 ligand 2 [Mus musculus]; Pitx1, paired-like homeodomain transcription factor 1 [Mus musculus]; POU5F1/OCT3, POU class 5 homeobox 1 [Homo sapiens]; Pou5f1/Oct3/4, POU domain, class 5, transcription factor 1 [Mus musculus]; Ppia, peptidylprolyl isomerase A [Mus musculus]; PROM1/CD133, prominin 1 [Mus musculus, Homo sapiens]; Rcor2, REST corepressor 2 [Mus musculus]; REST, RE1-silencing transcription factor [Mus musculus]; RFX, regulatory factor X-associated protein [Mus musculus]; Ripk3, receptor-interacting serine-threonine kinase 3 [Mus musculus]; RUNX1, runt related transcription factor 1 [Mus musculus]; Serpin/Serpinb2, serine (or cysteine) peptidase inhibitor, clade B, member 2 [Mus musculus]; Slc6a6, solute carrier family 6 (neurotransmitter transporter, taurine), member 6 [Mus musculus]; Sna1, snail family zinc finger 1 [Mus musculus]; Sox2, SRY (sex determining region Y)box 2 [Mus musculus]; SOX2, SRY-box transcription factor 2 [Homo sapiens]; Spire1, spire type actin nucleation factor 1 [Mus musculus]; STAT1, signal transducer and activator of transcription 1 [Homo sapiens]; Sting1, stimulator of interferon response cGAMP interactor 1 [Mus musculus]; Tap1, transporter 1, ATP-binding cassette, sub-family B (MDR/TAP) [Mus musculus]; Tap2, transporter 2, ATP-binding cassette, sub-family B (MDR/TAP) [Mus musculus]; Tbx4, T box 4 [Mus musculus]; TEAD, TEA domain family member 1 [Mus musculus]; Tfcp2, transcription factor CP2 [Mus musculus]; Ticam1/TRIF, toll-like receptor adaptor molecule 1 [Mus musculus]; TIr3, toll-like receptor 3 [Mus musculus]; TIr5, toll-like receptor 5 [Mus musculus]; Trpm4, transient receptor potential cation channel, subfamily M, member 4 [Mus musculus]; Ttll7, tubulin tyrosine ligase-like family, member 7 [Mus musculus]; Twist1, twist basic helix-loop-helix transcription factor 1 [Mus musculus]; Uba7, ubiquitin-like modifier activating enzyme [Mus musculus]; Vim, vimentin [Mus musculus]; Wee1, WEE 1 homolog 1 (S. pombe) [Mus musculus]; ZBTB, Zinc finger and BTB domain-containing protein [Mus musculus]; Zeb1, zinc finger E-box binding homeobox 1 [Mus musculus].

\section{Figures}


a
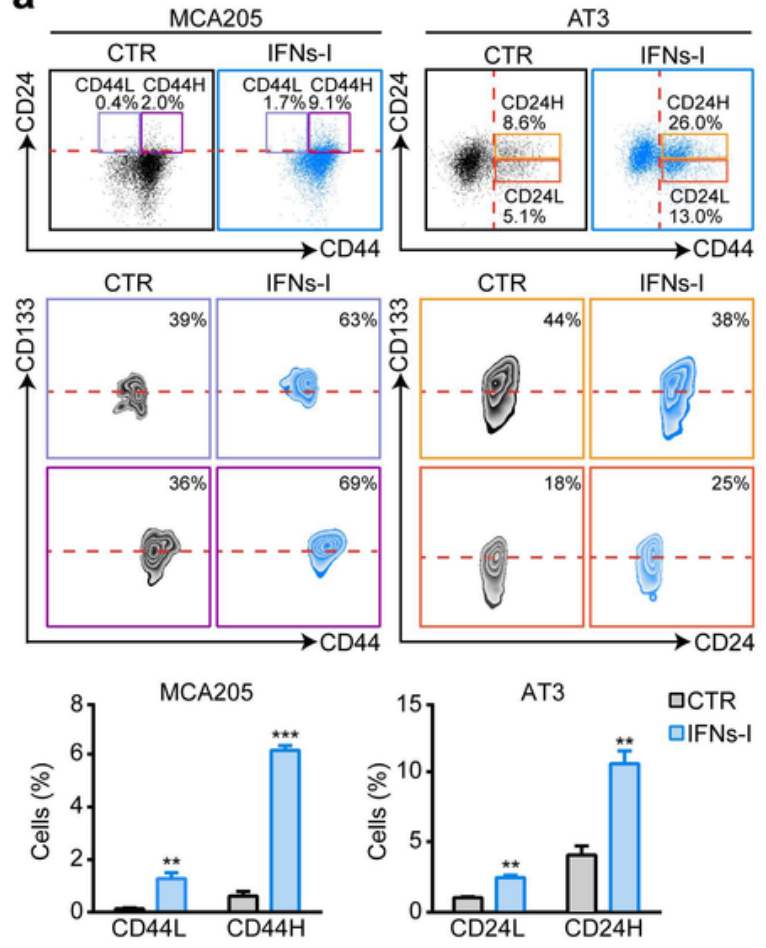

C
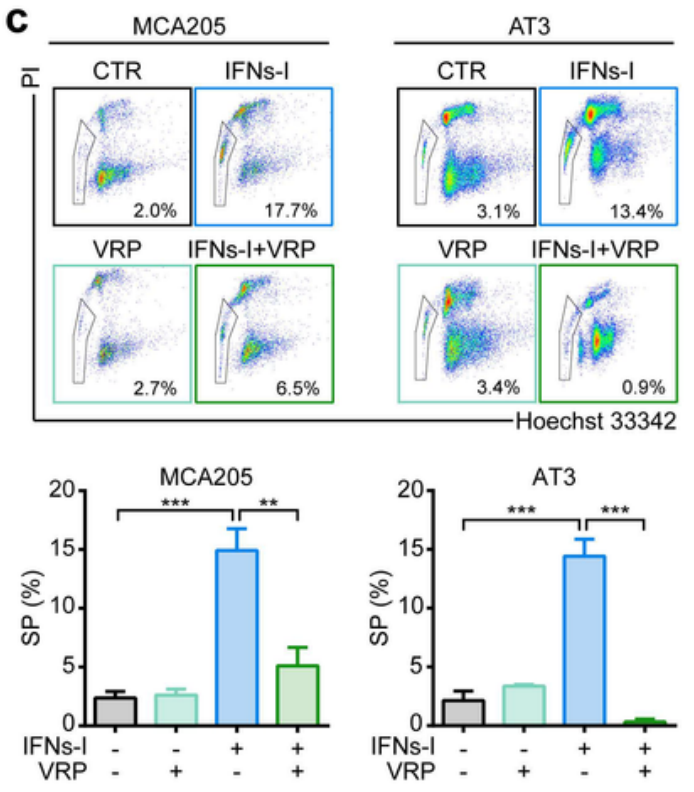

b

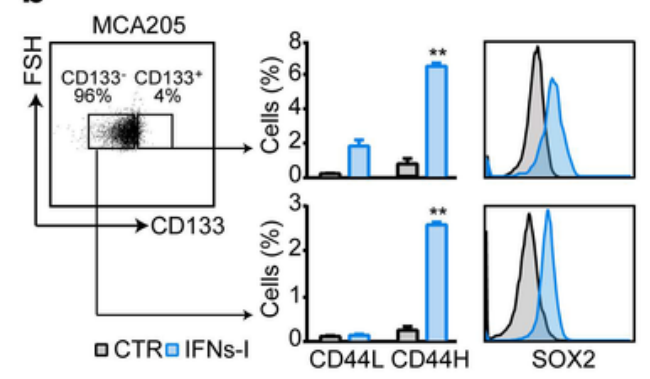

FC:

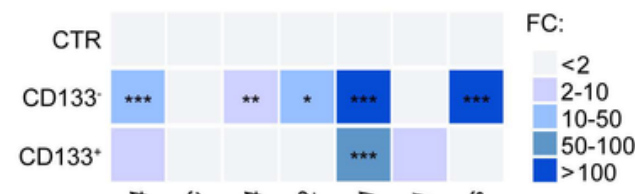

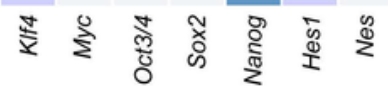

d

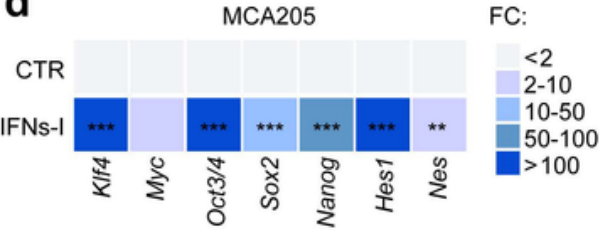

e
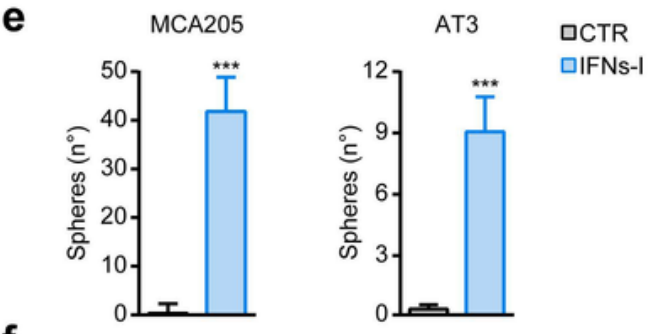

f
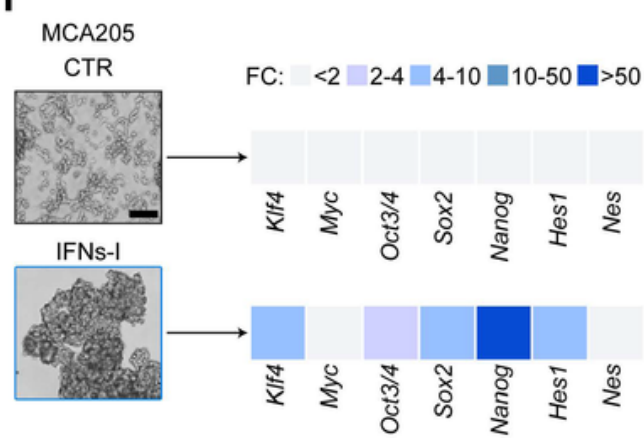

Figure 1

\section{Figure 1}

CSC appearance following IFN-I treatment. (a) Multiparametric flow cytometry analysis of the illustrated CSC surface markers in MCA205 and AT3 murine cell lines treated with mock (CTR) or purified IFNs-I $(6000 \mathrm{U} / \mathrm{mL}, 72 \mathrm{~h})$. Representative biparametric plots and a histogram showing the percentage (mean $\pm S E M ; n \geq 3$ ) of CD133+CD24+CD44+ cells obtained by calculating CD133+ cells within CD24+CD44+ subsets are shown. (b) Flow cytometry and qRT-PCR analyses of the reported CSC markers 
and reprogramming factors in FACS-isolated CD133- and CD133+ MCA205 cells, mock- or IFN-I-treated. One representative experiment out of three is shown. qRT-PCR data are reported as mean FC over untreated condition after intrasample normalization to Ppia expression levels. (c) Flow cytometry analysis showing the proportion of SP (Hoechst 33342- within PI-) cells in MCA205 ( $n \geq 3)$ and AT3 ( $n=3$ ) cells left untreated (black) or treated with VRP $(100 \mu \mathrm{M}$, light green), IFNs-I (blue) or VRP+IFNs-I (dark green). Data are presented as mean $\pm S E M$. (d) qRT-PCR analysis of the expression levels of the illustrated reprogramming factors in MCA205 cells treated with IFNs-I $(6000 \mathrm{U} / \mathrm{mL}, 72 \mathrm{~h})$. Data $(n \geq 3)$ are reported as in b. (e) Clonogenic potential of MCA205 and AT3 cells mock- or IFN--treated and plated in soft-agar. The histograms represent the number of spheres (mean $\pm S E M ; n=10$ ) generated, while representative images (f) show the capability of soft-agar-recovered IFN-I-treated MCA205 cells to grow as 3-D spheres in standard CSC culture conditions and to maintain a CSC-like transcriptomic profile. Scale bar $=100 \mu \mathrm{m}$. ${ }^{\star} \mathrm{P}<0.05,{ }^{*} \mathrm{P}<0.01,{ }^{*} * \mathrm{P}<0.001(\mathrm{a}, \mathrm{b}, \mathrm{d}, \mathrm{e})$ unpaired $\mathrm{t}$ test with Welch's correction, (c) ordinary one-way ANOVA test followed by Bonferroni's correction. See also Supplementary Figure 1. CD24H, CD133+CD44+CD24+high; CD24L, CD133+CD44+CD24+low; CD44H, CD133+CD24+CD44+high; CD44L, CD133+CD24+CD44+low; CSC, cancer stem cell; CTR, control; FC, fold change; IFNs-I, Type I interferons; PI, propidium iodide; SP, side population; VRP, verapamil. 
a

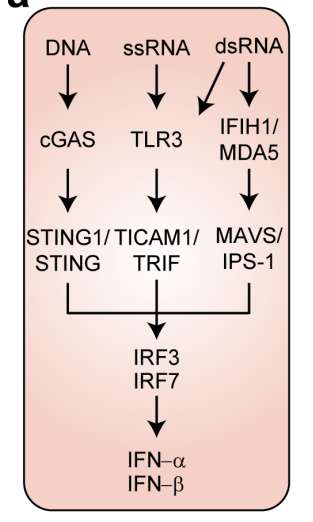

b
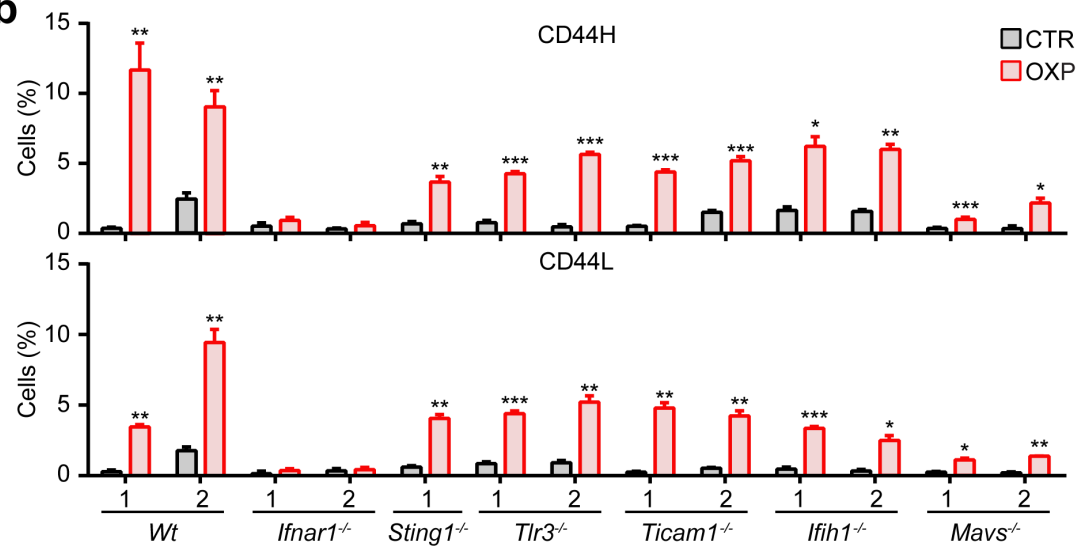

C

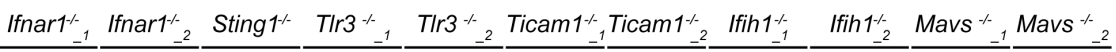

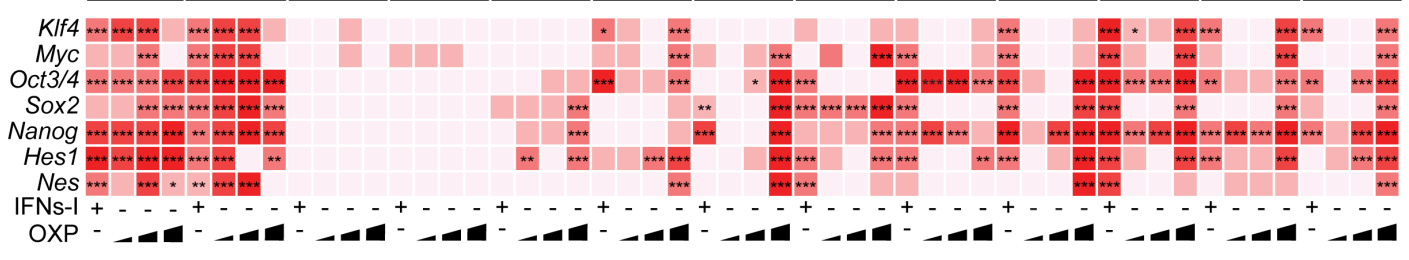

FC: $\quad<2 \square 2-10 \square 10-100 \square 100-1,000 \square>1,000$

d

AT3

e

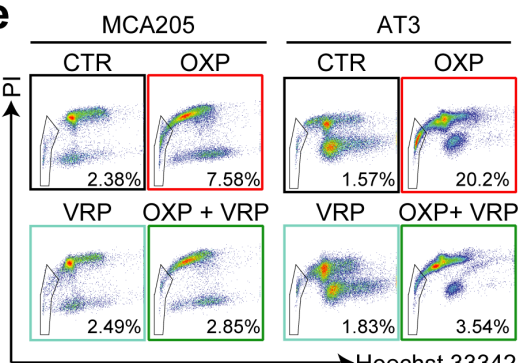

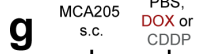

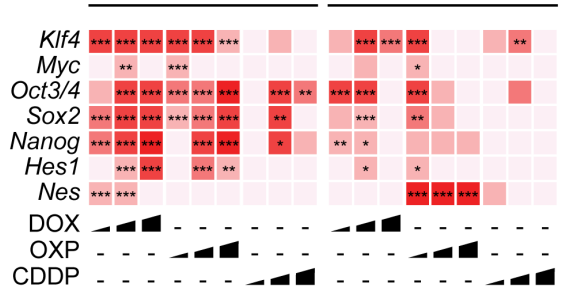

FC: $<2 \quad 2-5 \square 5-10 \square 10-100 \square>100$
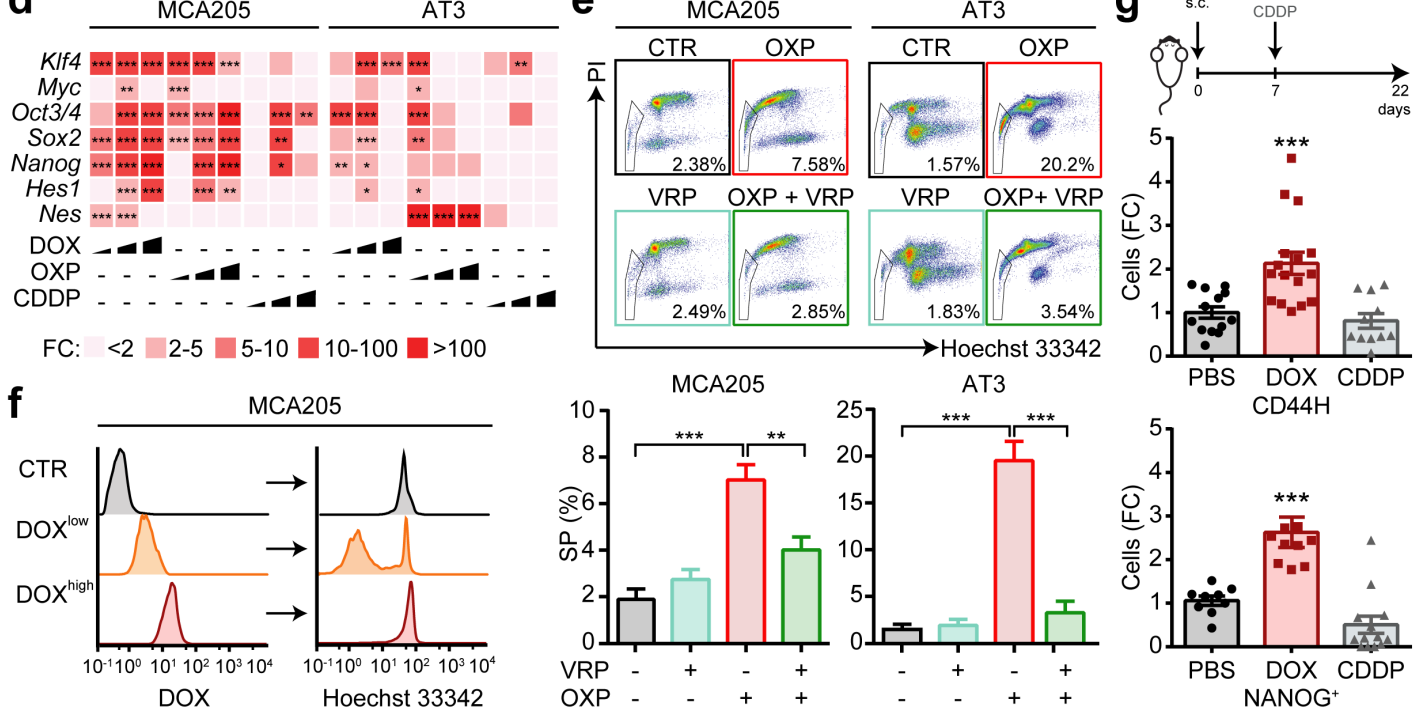

Figure 2

\section{Figure 2}

CSC promotion by immunogenic chemotherapy. (a) Major intracellular pathways upstream of IFNs-I. (b) Multiparametric flow cytometry analysis of CSC surface markers in MCA205-derived clones with the indicated genotypes left untreated (CTR) or treated with OXP $(300 \mu \mathrm{M}, 48 \mathrm{~h})$. The histograms represent the percentage (mean $\pm S E M ; n \geq 3$ ) of $C D 44 \mathrm{H}$ and CD44L cells. (c,d) Quantification by qRT-PCR of the expression levels of the illustrated reprogramming factors in MCA205 clones left untreated or exposed to 
$\operatorname{OXP}(3,30,300 \mu \mathrm{M}, 48 \mathrm{~h})$ or IFNs-I $(6000 \mathrm{U} / \mathrm{mL})(\mathrm{c})$ and in MCA205 and AT3 cells left untreated or administered with $\operatorname{DOX}(0.25,2.5,25 \mu \mathrm{M}), \operatorname{OXP}(3,30,300 \mu \mathrm{M})$, or $\operatorname{CDDP}(1.5,15,150 \mu \mathrm{M})(\mathrm{d})$. Data are reported as mean $F C$ over untreated condition ( $n \geq 2$ in $c$ and $n \geq 3$ in $d$ ) after intrasample normalization to the expression levels of Ppia. (e) Cytofluorometric evaluation of the proportion SP cells (Hoechst 33342within PI- cells) upon treatment of MCA205 and AT3 cells with OXP in the presence or not of VRP (100 $\mu M)$. Representative plots and quantitative analysis of the percentage of SP cells (mean $\pm S E M ; n \geq 3$ ) are shown. (f) Flow cytometry analysis of DOX efflux ability in MCA205 cells left untreated (in grey) or exposed to DOX $(2.5 \mu \mathrm{M}, 48 \mathrm{~h})$. The two DOXlow (orange) and DOXhigh (red) DOX-treated cell subsets display high and low capability to efflux DOX and Hoechst 33342. (g) Ex vivo flow cytometric analysis of CSC surface markers and intracellular NANOG expression in MCA205 cells grown in C57BL/6J mice treated intratumorally with vehicle (PBS) or $2.9 \mathrm{mg} / \mathrm{kg}$ DOX or $2.5 \mathrm{mg} / \mathrm{kg}$ CDDP ( $\mathrm{n} \geq 9 \mathrm{mice} / \mathrm{group}$ ). Data are presented as mean FC \pm SEM over PBS treatment. ${ }^{*} P<0.05$, ${ }^{\star \star} P<0.01$, ${ }^{\star *} * \mathrm{P}<0.001(\mathrm{~b}, \mathrm{~g})$ unpaired $t$ test with Welch's correction and Mann-Whitney test, (c) ordinary two-way ANOVA test followed by Bonferroni's correction $(\mathrm{d}, \mathrm{e})$ ordinary one-way ANOVA test followed by Bonferroni's correction. See also Supplementary Figure 2. CD44H, CD133+CD24+CD44+high; CD44L, CD133+CD24+CD44+low; CDDP, cisplatin; CSC, cancer stem cell; CTR, control; DOX, doxorubicin; FC, fold change; IFNs-I, Type I interferons; OXP, oxaliplatin; PBS, phosphate buffered saline; PI, propidium iodide; SP, side population; VRP, verapamil. 


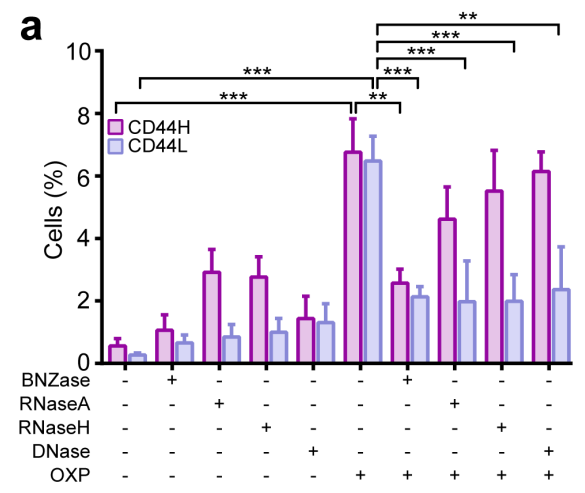

d

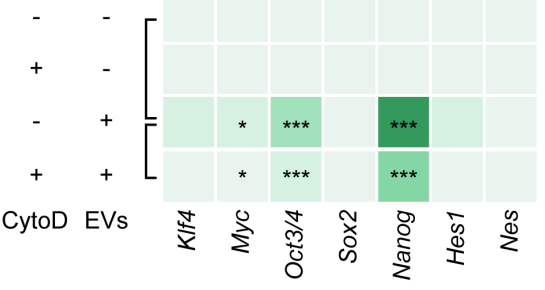

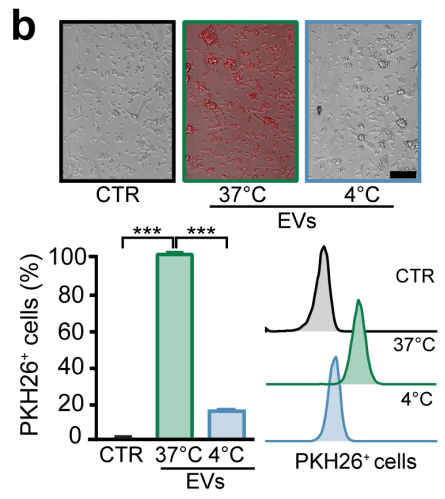

C

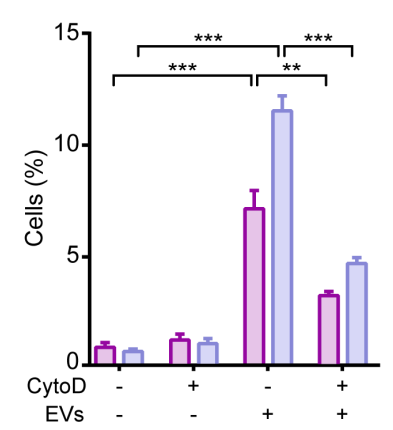

e

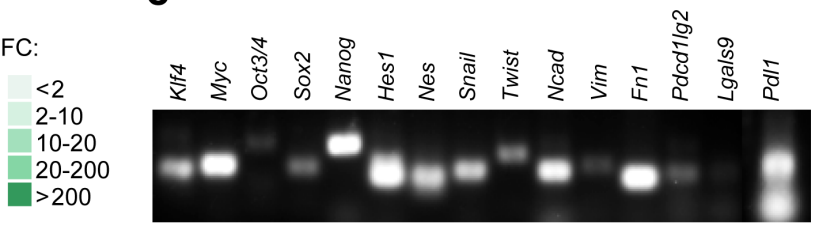

Figure 3

\section{Figure 3}

Cell-to-cell horizontal transfer of nucleic acids and de-differentiating factors during immunogenic chemotherapy. (a) Multiparametric flow cytometry analysis of CSC surface markers in "receiving" viable MCA205 cells upon co-culturing with "donor" MCA205 cells left untreated or previously treated with OXP $(300 \mu \mathrm{M}, 48 \mathrm{~h})$ alone or in combination with the indicated nucleases. Columns represent the percentage of CD44H and CD44L MCA205 cells, expressed as mean \pm SEM ( $n \geq 3$ ). (b) Fluorescence microscopy (upper 
panel) or flow cytometry (lower panel) analysis of the internalization (at $37^{\circ} \mathrm{C}$ and $4^{\circ} \mathrm{C}$ ) of donor cellderived, PKH26-stained EVs by “receiving” MCA205 cells. Scale bar: $100 \mu \mathrm{m}$. One representative experiment out of two is shown. (c) Multiparametric flow cytometry analysis of CSC surface markers in "receiving" MCA205 cells co-cultured with "donor" MCA205 cell-derived EVs in the presence of cyto D (0.5 $\mu M)$. Data are presented as mean $\pm S E M(n \geq 3)$. (d) Assessment of the expression levels of the indicated reprogramming factors by qRT-PCR in "receiving" MCA205 cells stimulated with "donor" MCA205 cellderived EVs alone or in the presence of cyto $D$, as described in c. Data are reported as mean FC $\pm S E M$ over control conditions of at least three independent experiments after intrasample normalization to Ppia expression levels. (e) Characterization of EV cargo by PCR analysis of a panel of the reported genes. ${ }^{*} \mathrm{P}<0.05$, ${ }^{* *} \mathrm{P}<0.01,{ }^{* * *} \mathrm{P}<0.001$ (a-d) one-way ANOVA test followed by Bonferroni's correction. See also Supplementary Figure 3. BNZase, benzonase; CD44H, CD133+CD24+CD44+high; CD44L, CD133+CD24+CD44+low; CSC, cancer stem cell; CTR, control; cyto D, cytochalasin D; EV, extracellular vesicles; FC, fold change; ICD, immunogenic cell death; OXP, oxaliplatin. 
a

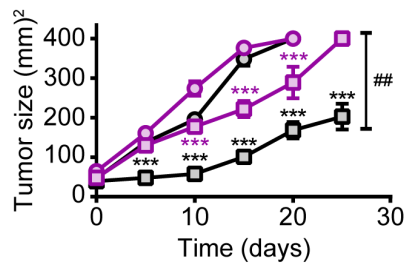

- Parental PBS - Parental DOX -O-CD44H PBS

C

\begin{tabular}{|c|c|c|c|c|}
\hline \multicolumn{5}{|c|}{ Cells } \\
\hline & $10^{2}$ & $0 \%$ & $0 \%$ & $0 \%$ \\
\hline & $10^{3}$ & $0 \%$ & $0 \%$ & $0 \%$ \\
\hline & $10^{4}$ & $0 \%$ & $0 \%$ & $0 \%$ \\
\hline & $10^{5}$ & $66 \%$ & $100 \%$ & $0 \%$ \\
\hline
\end{tabular}

\begin{tabular}{|c|c|c|c|c|}
\hline \multirow{5}{*}{ 黛 } & Cells & $\square$ & $\square$ & $\square$ \\
\hline & $10^{2}$ & $50 \%$ & $33 \%$ & $0 \%$ \\
\hline & $10^{3}$ & $50 \%$ & $100 \%$ & $33 \%$ \\
\hline & $10^{4}$ & $100 \%$ & $100 \%$ & $66 \%$ \\
\hline & $10^{5}$ & $100 \%$ & $100 \%$ & $100 \%$ \\
\hline
\end{tabular}

b

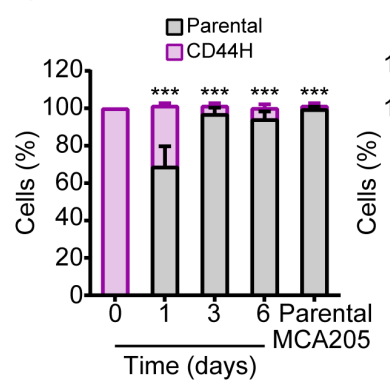

d
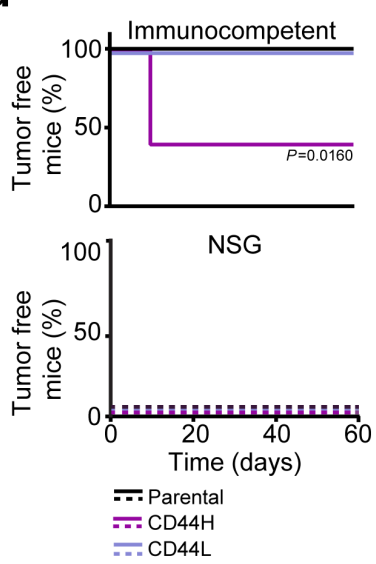

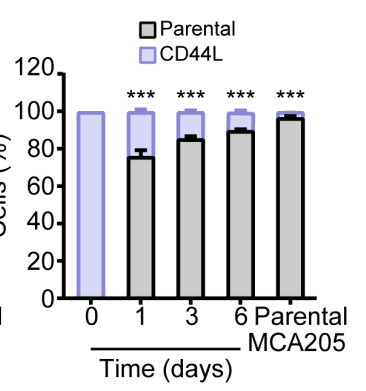

e

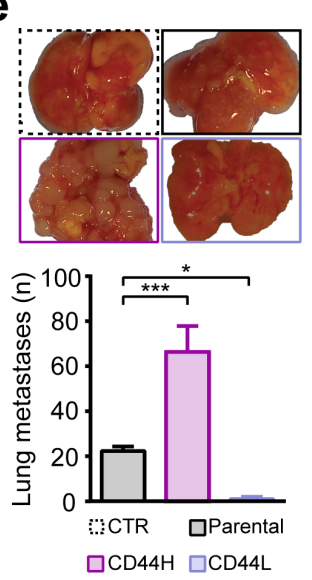

Figure 4

\section{Figure 4}

Functional characterization of ICD-CSCs. (a) Tumor growth of parental and CD44H MCA205 cells in C57BL/6J mice ( $\mathrm{n}=6 \mathrm{mice} / \mathrm{group})$, treated either with PBS or DOX $(2.9 \mathrm{mg} / \mathrm{kg})$. Growth curves show the mean tumor surface \pm SEM in one representative experiment out of two. (b) Flow cytometry analysis of the regenerative potential of FACS-isolated CD44H and CD44L MCA205 cells upon OXP treatment followed by cultivation in standard conditions for the indicated time. The histograms report the change in the 
percentage of ICD-CSCs evaluated by flow cytometry. (c,d) In vivo evaluation of the tumorigenic (c) and prophylactic (d) potential of parental MCA205 and ICD-CSCs upon inoculation in immunocompetent $\mathrm{C} 57 \mathrm{BI} / 6 \mathrm{~J}$ or immunodeficient NSG mice at the indicated dose ( $\mathrm{n}=3$ mice/group). In $\mathrm{d}, 1 \times 105$ parental MCA205 cells were injected in the other flank of immunocompetent or immunodeficient mice of $c$ that rejected the first injection. The percentage of tumor-free mice was the endpoint analyzed. (e) In vivo evaluation of the metastatic potential of parental MCA205 or ICD-CSCs injected via the tail vein of immunocompetent C57BI/6J mice ( $\mathrm{n}=3$ mice/group). Representative macroscopic observation of lung metastases 15 days post injection and quantification of the number of lung metastases in one representative experiment out of two are reported. ${ }^{*} \mathrm{P}<0.05,{ }^{*} \mathrm{P}<0.01,{ }^{\star} * \star \mathrm{P}<0.001(\mathrm{a}, \mathrm{b})$ two-way $\mathrm{RM}$ ANOVA (a) and ordinary two-way ANOVA (b) tests followed by Bonferroni's correction and unpaired t test with Welch's correction (d) Log-rank Mantel-Cox test (e) one-way ANOVA test followed by Bonferroni's correction. See also Supplementary Figure 4. CD44H, CD133+CD24+CD44+high; CD44L, CD133+CD24+CD44+low; CSC, cancer stem cell; DOX, doxorubicin; IFNs-I, Type I IFNs; MTX, mitoxantrone; NSG, NOD SCID gamma; OXP, oxaliplatin; PBS, phosphate buffered saline. 
a

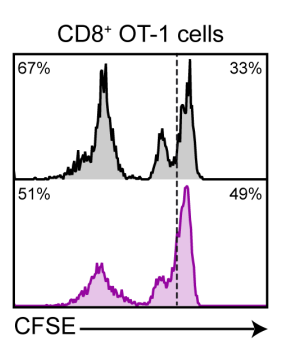

C
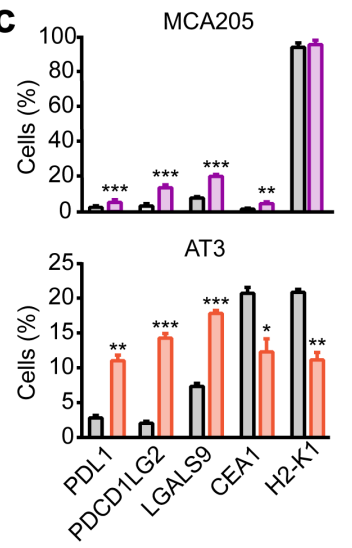

$\square$ Parental $\square \mathrm{CD} 44 \mathrm{H} \square \mathrm{CD} 24 \mathrm{~L}$

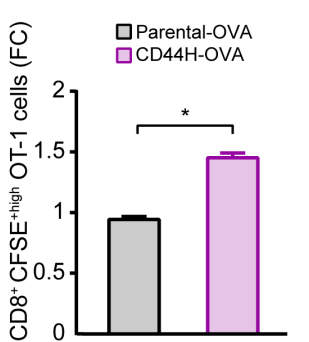

d
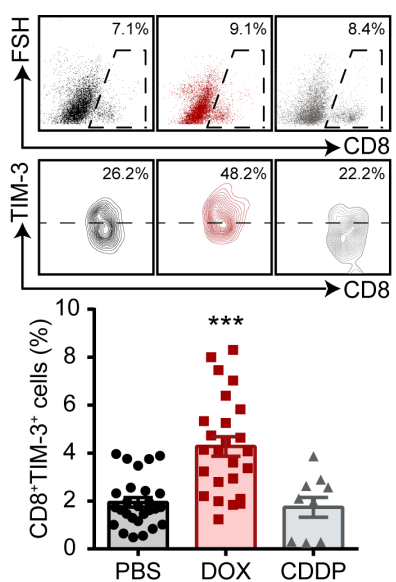

b
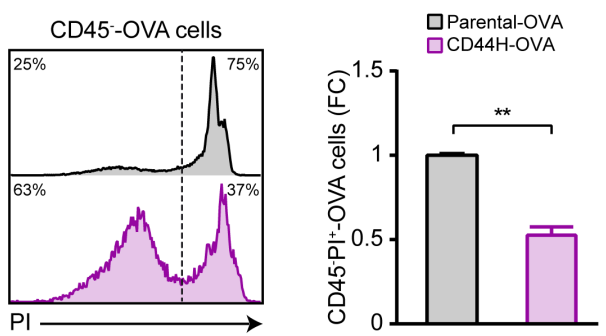

e

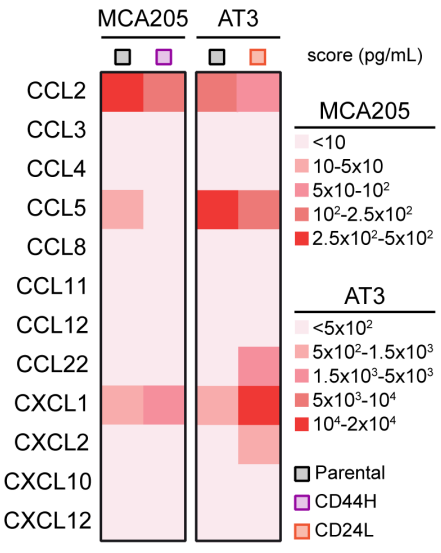

Distance $(\mathrm{mm})$

f
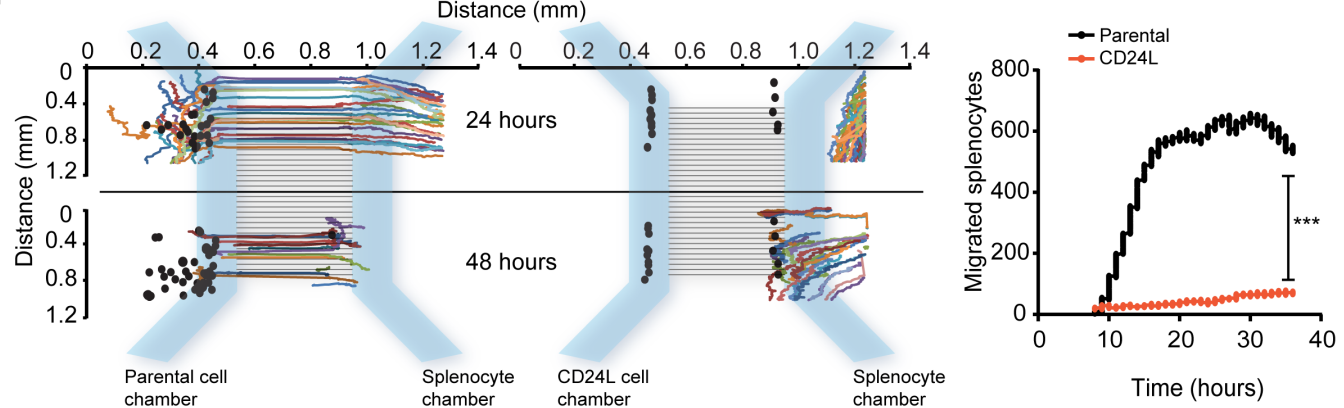

g

h
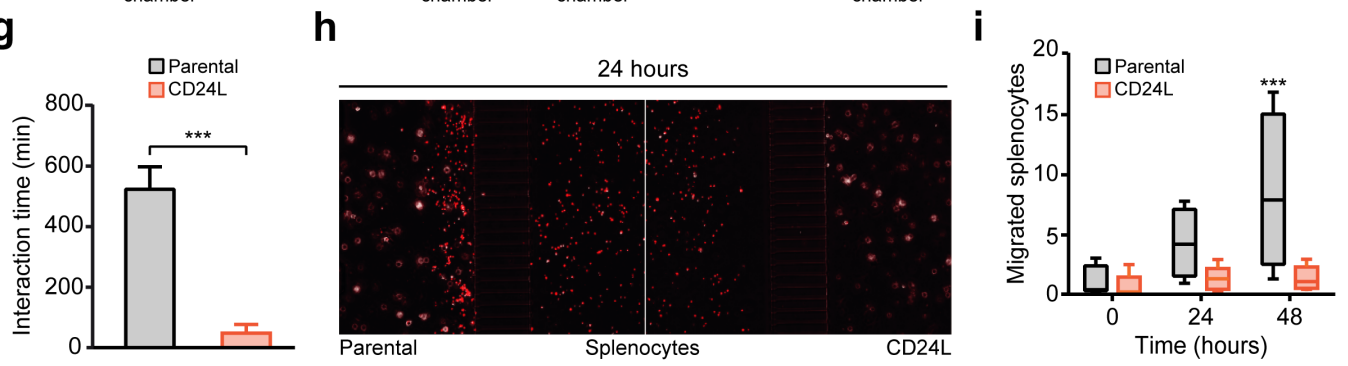

Figure 5

\section{Figure 5}

Phenotypic and functional profiling of IFN-CSC immunogenicity. (a) Flow cytometry analysis of the proliferation rate of CFSE-stained CD8+ OT-1 T cells stimulated with OVA-expressing parental or CD44H cells. The histograms represent the FC (mean $\pm S E M ; n=3)$ of not-proliferating CFSE+highCD8+ cells. (b) Flow cytometry analysis of the ability of CD45- OVA-expressing parental and CD44H cells to resist CD8+ OT-1-mediated killing. The histograms represent the FC (mean \pm SEM; $n=3$ ) of dying PI+CD45- cells. (c) 
Multiparametric flow cytometry analysis of the indicated IC molecules in MCA205 or AT3 cells (parental and ICD-CSCs). Data are presented as mean \pm SEM with $n \geq 3$. (d) Flow cytometry analysis of TIM-3 expression in CD8+ TILs from MCA205-derived xenografts 15 days post in vivo intratumoral treatment with PBS, or DOX (2.9 mg/kg), or CDDP (2.5 mg/kg) ( $\mathrm{n} \geq 10$ mice/group). Data are presented as mean $\pm S E M$ of three independent experiments. (e) Quantification of released chemokines in supernatants from MCA205 and AT3 cells (parental and ICD-CSCs) by Luminex Multiplex Assay. One representative experiment out of two is shown. (f-i) Time-lapse analysis of the migration of $\mathrm{H}-2 \mathrm{~Kb}$ splenocytes towards parental and CD24L AT3 cells in microfluidic devices. Plots representing the trajectories of individual splenocytes towards target cancer cells (black spots) recorded in time-lapse are reported in $f(n=820)$, while quantification of the interaction times between splenocytes and parental or CD24L ICD-CSCs are shown in $\mathrm{g}$ ( $\mathrm{n} \geq 10$ cells per condition analyzed; see also Supplementary Movies 1-4). Representative pictures of splenocytes in competition microfluidic devices (scale bar: $100 \mu \mathrm{m}$ ) and quantification of splenocytes migrated towards parental or CD24L ICD-CSCs are shown in h and i, respectively. Data are expressed as mean \pm SEM $(n \geq 4) .{ }^{*} P<0.05,{ }^{*} P<0.01,{ }^{*} * P<0.001$ (a-c, $\left.f\right)$ unpaired $t$ test with Welch's correction and one-way ANOVA test followed by Bonferroni's correction, $(\mathrm{d}, \mathrm{g})$ Mann-Whitney test, (i) twoway RM ANOVA test followed by Bonferroni's correction. See also Supplementary Figure 4 and Supplementary Movies 1-4. CD24L, CD133+CD44+CD24+low; CD44H, CD133+CD24+CD44+high; CDDP, cisplatin; CFSE, carboxyfluorescein succinimidyl ester; CSC, cancer stem cell; DOX, doxorubicin; FC, fold change; ICs, immune checkpoints; ICD-CSCs, immunogenic cell death-induced cancer stem cells; OVA, ovalbumin; PBS, phosphate buffered saline; TILs, tumor infiltrating lymphocytes. 


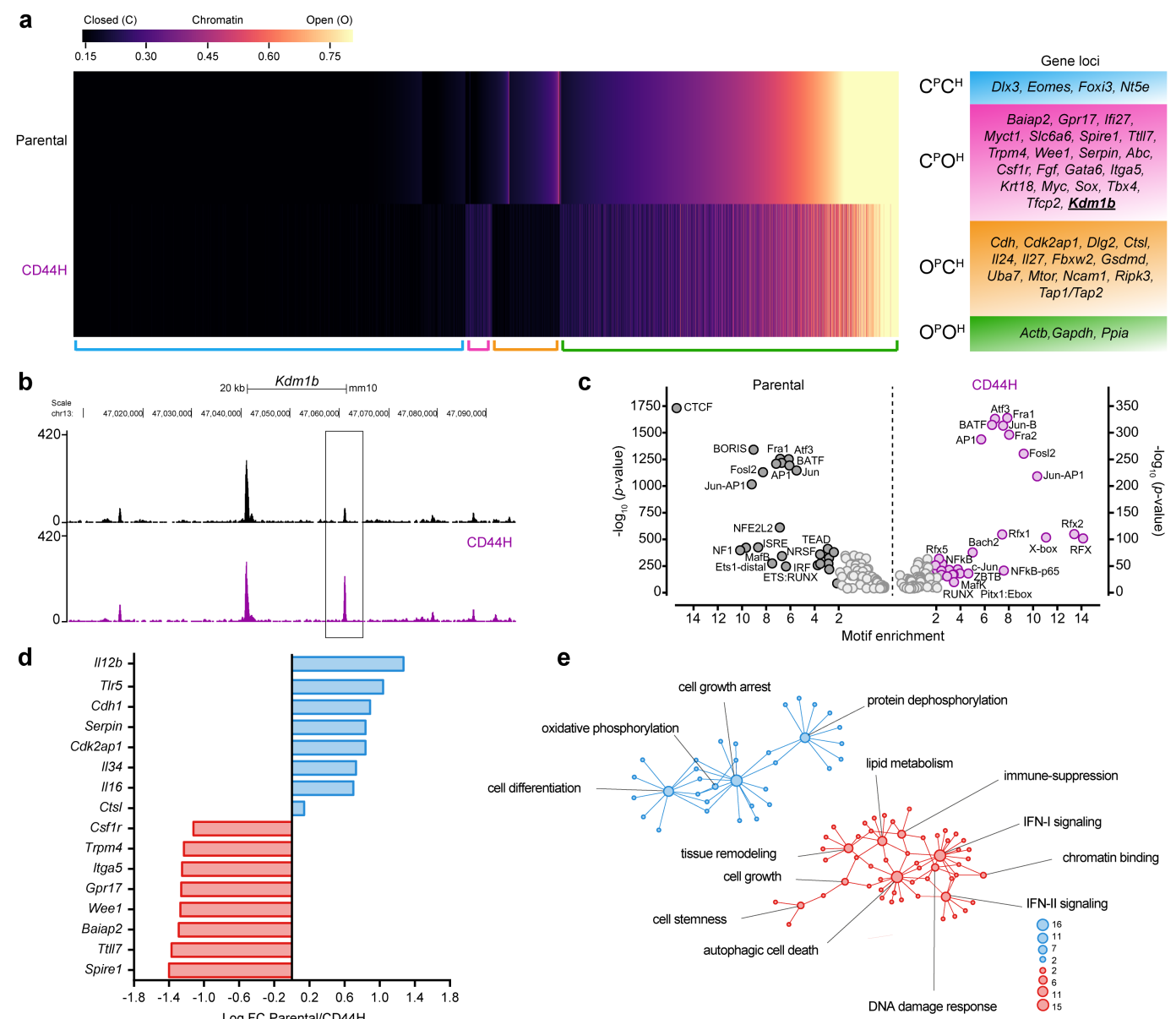

f

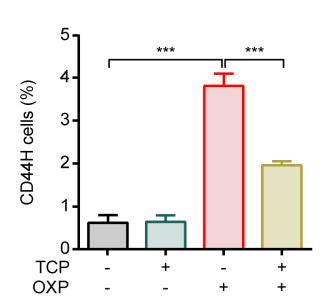

g

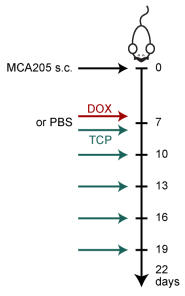

C
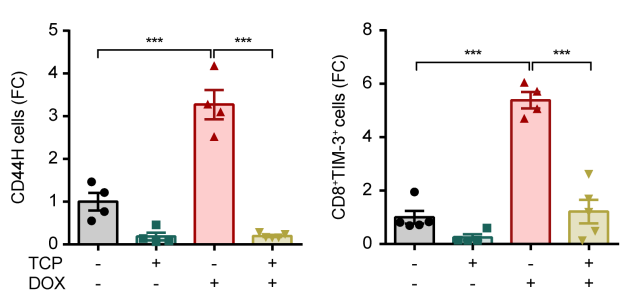

n

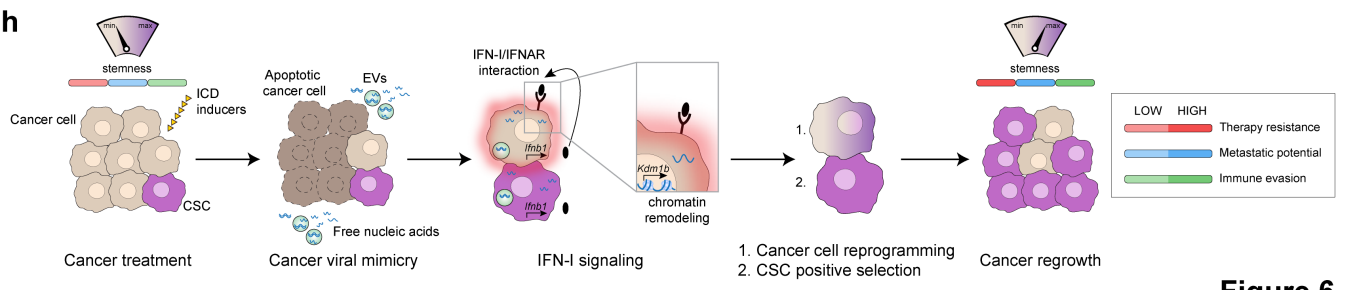

Figure 6

\section{Figure 6}

IFN-I-driven chromatin remodeling. (a-e) ATAC-seq (a-c) and RNA-seq (d,e) analysis in parental or FACSisolated CD44H MCA205 cells upon enrichment via IFN-I administration $(6000 \mathrm{U} / \mathrm{mL}, 72 \mathrm{~h})$. The heatmap in a illustrates global open $(O)$ or closed $(C)$ status of genes in parental $(P)$ and $C D 44 H(H)$ cells and gene stratification in 4 subgroups according to chromatin accessibility profiles: $\mathrm{C}$ or $\mathrm{O}$ in all samples $(\mathrm{CPCH})$ and $(\mathrm{OPOH})$, and differentially $\mathrm{O}$ or $\mathrm{C}$ in the two cell subsets ( $\mathrm{CPOH}$ and $\mathrm{OPCH})$. Representative genes for 
each subgroup are shown. In b, representative loci for $\mathrm{Kdm} 1 \mathrm{~b}$ within $\mathrm{CPOH}$ group are illustrated. (c) TF family binding motifs enriched at least 2-fold in loci more accessible in parental (black) or CD $44 \mathrm{H}$ (purple) cells; the $x$ axis shows the log FC of TF motif enrichment in target cells (either parental or $\mathrm{CD} 44 \mathrm{H}$ ) over non-target cells; the y axis depicts the significance level of enrichment. (d) Patterns of gene expression as determined by RNA-seq for representative ATAC-seq-identified genes. (e) Genes upregulated (red) and downregulated (blue) in CD44H IFN-CSCs were used to generate a GO network analysis, visualized by using clusterProfiler and enrichPlot R packages. Nodes represent enriched GO-terms, whereas node size represents the corresponding FDR-adjusted enrichment $P$ value (q value). ( $f$ ) Multiparametric flow cytometry analysis showing the percentage of CD44H ICD-CSCs upon OXP (300 $\mu \mathrm{M}$, $48 \mathrm{~h})$ treatment alone or in combination with the KDM1B inhibitor TCP $(10 \mu \mathrm{M})$. Data are presented as mean \pm SEM $(n \geq 4)$. (g) Schematic experimental protocol of in vivo KDM1B inhibition (left panel) and multiparametric flow cytometry analysis showing the $\mathrm{FC}$ of $\mathrm{CD} 44 \mathrm{H}$ (central panel) and CD8+TIM-3+ (right panel) cells in tumoral bulks from C57BI/6J mice treated with DOX $(2.9 \mathrm{mg} / \mathrm{Kg})$ in combination or not with TCP $(5 \mathrm{mg} / \mathrm{Kg})$ ( $\mathrm{n} \geq 4$ mice/group). (h) Graphical summary. ${ }^{* *} \mathrm{P}<0.001$ (ordinary one-way ANOVA test followed by Bonferroni's correction). See also Supplementary Figure 5. CD44H, CD133+CD24+CD44+high; FC, fold change; FDR, false discovery rate; GO, gene ontology; ICD-CSCs, immunogenic cell death-induced cancer stem cells; IFNs-I, Type I IFNs; TCP, tranylcypromine; TF, transcriptional factor. 
a
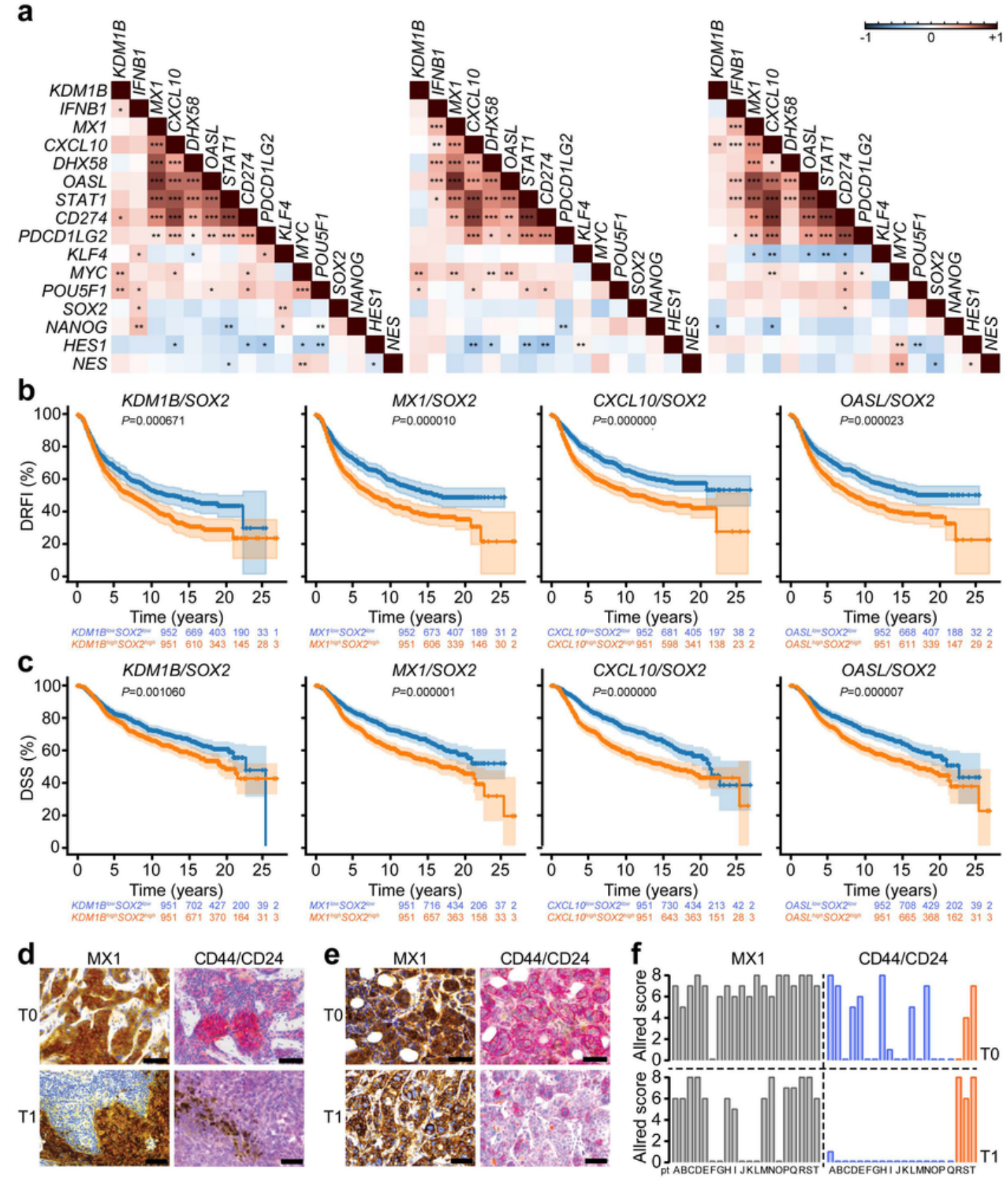

Figure 7

\section{Figure 7}

Correlation between KDM1B, IFN-I signature, stemness and immune evasion in BC patients. (a) Spearman correlations between KDM1B, IFN-I-related, CSC-related and IC-related gene expression scores from microarray data of three publicly available cohorts of $\mathrm{BC}$ patients treated with neo-adjuvant anthracycline-based chemotherapy. ${ }^{*} P<0.05$, ${ }^{* *} P<0.01$, ${ }^{* * *} P<0.001$. (b,c) Kaplan-Meier plots depicting DRFI (b) and DSS (c) in BC patients stratified according to risk behaviour via the Metabric platform 
(https://ega-archive.org/) and expressing low (blue curves) or high (orange curves) levels of SOX2 (GenBank/Entrez ID: 6657) and either KDM1B (GenBank/Entrez ID: 221656), or MX1 (GenBank/Entrez ID: 4599), or CXCL10 (GenBank/Entrez ID: 3627), or OASL (GenBank/Entrez ID: 8638). P Cox, Log-Rank (Mentel-Cox) $P$ value was calculated using the Metabric website. $P$ values $₫ 0.05$ were considered statistically significant. (d-f) IHC analysis of 20 paraffin-embedded paired BC biopsies at T0 (diagnosis) and T1 (surgery) using antibodies directed against MX1, CD45 and CD44+CD24. Representative IHC images from sections of two patients are reported in $\mathrm{d}$ and e (scale bar $=30 \mu \mathrm{m}$ ), while Allred MX1 and CD44+CD24+low/- scores for 20 patients at T0 and T1 are shown in $\mathrm{f}$. In f, orange hystograms represent patients showing an increase of CSCs after therapy. See also Supplementary Figure 6. BC, breast carcinoma; CSCs, cancer stem cells; DRFI, distant relapse free incidence; DSS, disease specific survival; ICs, immune checkpoints; IFNs-I, Type I IFNs; IHC, immunohistochemistry.

\section{Supplementary Files}

This is a list of supplementary files associated with this preprint. Click to download.

- SupplementaryMovie1MusellaetalNI.avi

- SupplementaryMovie2MusellaetaINI.avi

- SupplementaryMovie3MusellaetalNI.avi

- SupplementaryMovie4MusellaetaINI.avi

- SupplementaryInformationMusellaetal2021.pdf 